\title{
PROGRESS REPOFT
}

\author{
U.S. DEPARTMENT OF ENERGY CONTRACT \\ DE-AS-05-76ER05096, High Energy
}

$$
1983-1984
$$

\section{RICE UIJIVERSITY}

T. W. Bonner Nuclear Labcratories, Physics Department

G. C. Phillips, Paircipai Investigator

J. B. Roherts, Co-Principal Investigator

Houston, Texas

October 1, 1982 


\section{DISCLAIMER}

This report was prepared as an account of work sponsored by an agency of the United States Government. Neither the United States Government nor any agency Thereof, nor any of their employees, makes any warranty, express or implied, or assumes any legal liability or responsibility for the accuracy, completeness, or usefulness of any information, apparatus, product, or process disclosed, or represents that its use would not infringe privately owned rights. Reference herein to any specific commercial product, process, or service by trade name, trademark, manufacturer, or otherwise does not necessarily constitute or imply its endorsement, recommendation, or favoring by the United States Government or any agency thereof. The views and opinions of authors expressed herein do not necessarily state or reflect those of the United States Government or any agency thereof. 


\section{DISCLAIMER}

Portions of this document may be illegible in electronic image products. Images are produced from the best available original document. 


$$
\text { DOE/ER/05096--10 }
$$$$
\text { DE83 } 001019
$$

\section{HIGH - ENERGY PHYSICS}

\section{PPOGRESS REPORT}

\section{MASTER}

\section{Iorra}

\section{FORTIONS_OF THIS REPORT ARE ILLEGIBLE. It} chas been reproduced from the best avallablo cony to permit the broadest possible avall-
ability:

G. C. Phillips

J. B. Roberts

William Marsh Rice University

m. W. Bonner Nuclear Laboratories

March 1, 1982: - February 28, 198."

Prepared for the

U. S. DEFARTMENT OF ENERGY

D. O. E. Report $\# D O E / E P / 05096-10$ 


\section{ABSTRACT}

Considerable progress has been made in high energy physics research on several fronts during the present contract year. Analysis of the data from ZGS polarized beam experiments E-460 and E-462 has continued and is nearing completion. This, along with our data from LAMPF E-504/505, will have considerable bearing on the question of dibaryon resonances. Work has continued on the project to accelerate polarized proton beams in the Brookhaven AGS. One of our contributions to this project, the $200 \mathrm{MeV}$ polarimeter, will be completed, calibrated, and installed in the AGS LINAC during this contract year. The combined proposal E-704 for the first round polarized beam experiment at FNAL was approved and an agreement was reached among the various collaborators and submitted to the laboratory. The first run of the hadron jet calorimeter experiment, FNAI E-609, was completed during this contract year. Data analysis is underway which will constitute three Ph.D. theses for Rice students. We have also investigated additional future directions for the program, such as neutrino oscillation studies, high energy $e=p$ collisions, underground detectors, and experiments at LAMPF II. 
D.O.E. CONTRACT NO. DE-AS-05-76ERO5096

\section{PROGRESS REPORT}

T. W. Bonner Nuclear Laboratories Rice University, Houston, Texas

Personnel working on the project during the year to Feb. 28,1983

G. C. Phillips, Professor of Physics and Director, T.W.Bonner Nuclear Laboratories - Principal Investigator

J. B. Roberts, Associate Professor of Physics, Co-Prinipal Investigator

S. D. Baker, Professor of Physics

G. S. Mutchler, Professor of Physics

B. E.Bonner, Visiting Professor of Physics

H. E. Miettinen, Assistant Professor of Physics

M. D. Corcoran, Assistant Professor of Physics Minh Duong-Van (Ph.D., Cornell, 1973) Senior Research Associate 
M. M. Calkin (M.A., Rice University) Graduate Research Assistant

D. A. Bell (B.S., University of Texas Arlington) Graduate Research Assistant

K. A. Johns (B.A., Rice University) Graduate Research Assistant

J. A. Rice (M.A., Yale University) Graduate Research Assistant

M. R. Marcin (M.A., University of Massachusetts) Graduate Research Assistant

C. J. Naudet (B.S., University of Kansas) Graduate Research Assistant

J. A. Buchanan (B.S., University of Houston) Senior Research Engineer

J. M. Clement (Ph.D., Rensselaer Poly.Inst.) Research Engineer

Joe Windish, Technician

Jim Chappell, Scientific Instrument Maker

Helen Viereck (M.A., University of Houston) Administrative Assistant 
Table 1

PUBLICAIIONS, THESIS, TIN PPISENINATIONS

From High Energy Experiments Performed by the

Rice University ingh Energy Group

\section{COMPLETED ZGS EXPERIMENTS}

E-395:

Publications: 1. "Measurement of $\Delta \sigma_{\text {tot }}$ for $p \uparrow-p \uparrow$ at 1-3 GeV/c," Physics Letters 73F (1978) 235.

2. Measurement of the Energy Dependence

of $\Delta \sigma_{T}$ and ${ }^{A}{ }_{N N}$ for pp scattering in the $1-3 \mathrm{GeV} / \mathrm{C}$ Region," AIP Conf.Froc. ivo. 51, ed. G. H. Thomas (1979) p. 423.

3. Measurement of the pp Total Cross

Section in pure Transverse spin states in the 1-3

GeV/C Region," Bull. APS 25 (April 1980) 562.

4. "Transverse Spin Dependence of pp

Total Cross Sections," AIP Conf.Froc. No. 35 (1976)72.

E-407:

Publications: 1. "Polarization in pp Elastic Scattering at Small. $|t|, "$ Phys.Rev. 015 (1977) 602.

2. "Depolarization Parameter in pp Inclusive scattering at $6 \mathrm{GeV} / \mathrm{c}$," Phys.Rev.Lett. 44 (May 26, 1980) 1373-76.

E-393, E-408:

Publications: 1. "Measurement of Pion Asymmetries in Inclusive Proton-Proton Scattering," Phys.Rev.Lett. 36 (1976) 929.

2. "Asymmetries in Inclusive Proton-Nucleon Scattering at $11.75 \mathrm{GeV} / \mathrm{C}, "$ Phys.Rev. 018 (December 1978) 3939. 
E-393, E-108: 3. Measurement of Asymunetries in Inclusive Proton-proton scattering," AIP Conf. Proc. No. $35(1976) 219$.

E-415:

Publication: "Search for Direct Electron production in pp and $p-B e$ Collisions at $12 \mathrm{GeV} / \mathrm{c}, "$ Phys.Rev. Lett. 41 (1978) 367.

$E-418:$

Publication: "Analyzing power in Large-Angle pp and pn Elastic Scattering," phys.Rev.Lett. 45 (1980) 1529.

E-425:

Thesis: "Measurement of $A_{\text {TNN }}$ in $n-p$ Elastic Scattering at $6 \mathrm{GeV} / \mathrm{c}, "$ M.A. Thesis, Rice University, M. M. Calkin $(1930 \div 81)$.

Publication: "Measurement of Spin Parameter A and $A_{N N}$ in np and pp Elastic scattering at $6 \mathrm{GeV} / \mathrm{c}$," APS Bull. 26 (April 198I) 549.

E-434:

Publications: I. "Measurement of $A$ and $A_{N N}$ in $p-p$ Elastic Scattering from $1-3 \mathrm{GeV} / \mathrm{C}, "$ Phys.Lett. 94B (1980) 310.

2. "Energy Dependence of $A_{N N}\left(90^{\circ}\right)$ in $p p$ Elastic scattering from 1..10 to $2.75 \mathrm{GeV} / \mathrm{c}$," Polarization Phenomena in Nuclear Physics, Santa Fe-1980, AIP Conf.Proc. No. 69 (1981), p. 152.

3. "Measurement of the Spin Parameters A and $A_{N y}$ in pp Elastic scattering in the $1-3 \mathrm{GeV} / \mathrm{C}$ Region," APS Bull. 25 (April 1980) 562.

4. "Folarimeters: A Summary," AIP Conf. Proc. No. 42 (1978) 67 .

5. "ZGS Polarized Beam Program," AID Conf. Proc. No. 60 (1980) 158 . 
High Energy Puilications, Theses, \& Presentations

$E-434$

Publications: 6. "Evidence for Dibaryon Resonances in Nucleon-Uucleon Scattering," Polarization Phenomena in Nuclear Physics, Santa Fe-1980, AIP Conf.Proc. No. 69 (1981) P. 31.

E.-437:

Publications: 1. "Analyzing Power in the Reactions $p+d \rightarrow d+p$ and $p+p \rightarrow d+\pi$ at GeV Energies," phys.Rev.Lett. 41 (1978) 1098 .

2. "Polarization in Elastic $\mathrm{p}-\mathrm{p}$ and $\mathrm{p}-\mathrm{n}$ Scattering at $1.03 \mathrm{GeV}, "$ Phys.Rev. C18 (1978) 331.

E-445: "Asymmetries in Inclusive $\pi^{-}$Production with a Polarized Beam and Target;" these data have not been analyzed as yet.

$\mathrm{E}-460$ :

Theses: 1. "Measurement of the pp Total Cross Section in Pure Transverse Spin states in the 1-3 GeV/C Region," M.A. Thesis, Rice University, J. D. Lesikar (1980).

2. Measurement of the $\mathrm{pp}$ and pd Total Cross Sections in Pure Transverse spin states in the 1-3 GeV/C Region," Ph.D. Thesis, Rice University, J. D. Lesikar, August 21; 1981.

Publications: 1. "Z.GS Polarized Beam Program," AIP Conf.Proc. No. 60 (1980) 158.

2. Measurement of the $p p$ and pd Total Cross Section in Pure Transverse Spin states in the 1-3 GeV/C Region," APS Bull. 26 (April 1981) 549.

$E-462:$

publications: 1. "A Search for Narrow Resonances in $\mathrm{pp} \rightarrow \mathrm{p} \pi^{+} \mathrm{n}$ at $2 \mathrm{GeV} / \mathrm{c}, "$ High Energy Physics-1930, AIP Conf. Proc. No. 68 [formerly ANL-HEP-CD-80-26]. 
Hioh Energy Publications, Theses, and Presentations

E- $-462:$

Publications: 2. Analyzing Power in the Reaction $p p \rightarrow d \pi^{+}$ for Beam Momenta from 1.17 to $1.96 \mathrm{GeV} / \mathrm{c}$," submitted to Fhysical Review Comments.

3. "A High Statistics study of the Reaction $\mathrm{pp} \rightarrow \mathrm{p} \pi^{+} \mathrm{n}$ Between 1.2 and $2.0 \mathrm{GeV} / \mathrm{c}, " \mathrm{APS}$ Bull. 26 (April 1981) 549.

4. "Asymmetry in the Reaction $p \underline{p} \rightarrow \mathrm{d} \pi^{+}$ at Intermediate Energies," Ibid.

E-609:

Publications: "Evidence for Jets From a Transverse Energy Triggered Calorimeter Experiment at Fermilab," presented at XXI Conference on High Energy Physics, Paris, July, 1982 .

FUTURE EXPERIMENTS AND PROJECTS, PROPOSED OR APPROVED

E-559 (LAMPF) "Search for Neutrino Oscillations and Violation of Lepton Number Conservation." The proposal was rejected at the January 1982 PAC Meeting. It was submitted to D.O.E. as an instrumentation development project proposal.

E-609 (FNAL) "A Study of the structure of High $\mathrm{p}_{\mathrm{T}}$ Hadron Interactions," This experiment was run during this contract year. We are scheduled for Tevatron running in 1983-1984 as a higher energy continuation.

E-704 (FNAL) "First Round Experiments with the Polarized Beam Facility." This experiment was approved at the November 1981 PAC Meeting for 1000 hours of Tevatron time and is presently scheduled as a first round new Tevatron II project. Design studies for equipment will begin during this contract year. 
AGS Polarized Beam Acceleration Project: Construction. is completed on the $200 \mathrm{MeV}$ polarimeter and it will be calibrated at the Indiana Cyclotron Facility and then installed on the AGS Linac before the end of this contract year.

Publications: 1. "Polarized Protons at ISABELLE," Proceedings of the ISABELLE Summer Study; 1981. 2. "Report on e x p Experiments at ISABELLE: , Ibid. 


\section{PAGES 8 to 9 WERE INTENTIONALLY LEFT BLANK}


A. POIARIZATION STUDIES

During the past year we have continued to analyze data from our extensive use of the zGS polarized proton beams and the ANL/Rice polarized proton target and the ANL Effective . Mass spectrometer system. Two such efforts are reported below. In addition our ongoing efforts in two collaborations -- polarized beam development at BNI/AGS and at FNAL, are reported below.

1. Measurement of the pp and pd Total Cross section in Pure Transverse spin states in the 1-3 GeV Region [E-460, ZGS ] [J.D. Lesikar and J.B. Roberts]

The final corrections to the data of E-460, ZGS were completed this contract year and the pure-spin-state cross sections $\Delta \sigma_{T}$ for the $I=0$ initial state were extracted from the pp and pd measurements by J.D. Lesikar. The Coulomb-Nuclear interference corrections were recalculated using the most recent forward scattering amplitudes $F_{1}, F_{2}$, and $F_{3}$, for p-p scattering from the dispersion relation calculations of Grein and Kroll ${ }^{1}$. The $\Delta \sigma_{T}(p p)$ data is in good agreement with the data of Bystricky et al. on the shape of the energy dependence, but disagrees somewhat in the normalization. This data is, however, used for the $p-d, p-p$ subtraction since $\Delta \sigma_{T}(p d)$ is normalized in the same fashion. 
An iterative procedure was used to calculate the correction to the subtraction $\delta \sigma_{T}$ :

$$
\Delta \sigma_{T}(p n)=\frac{\Delta \sigma_{T}(p d)}{1-3 / 2 p d}-\Delta \sigma_{T}(p p)-\delta \sigma_{T}
$$

since the correction in itself involves $\Delta_{T}(p n)\left(F_{2}{ }^{p n}\right)$. The values of $F_{1}$ and $F_{2}$ for $p p$ and $p$ scattering from the analysis of Grein and Kroll were used to calculate $\delta \sigma_{T}$. The pp cross sections were corrected for smearing due to Fermi motion within the deuteron. The values of $\Delta \sigma_{T}(p n)$ converged to within the statistical errors after only three iterations. $\Delta \sigma_{T}(I=0)$ is plotted in Fig.l for $P_{L}$ of 1.2 to 2.5 $\mathrm{GeV} / \mathrm{c}$.

The shape of the cross section as a function of energy is in agreement with the earlier data based on less elaborate corrections. The absolute error window, due to lack of knowledge of the absolute deuteron target polarization, are shown as dashed lines. However, the relative errors on the energy dependence are much smaller, and show the broad peak centered at $1.4-1.5 \mathrm{GeV} / \mathrm{c}$ which has also been reported in $\Delta \sigma_{L}(I=0)$.

${ }^{1}$ w.grein, private communication. 


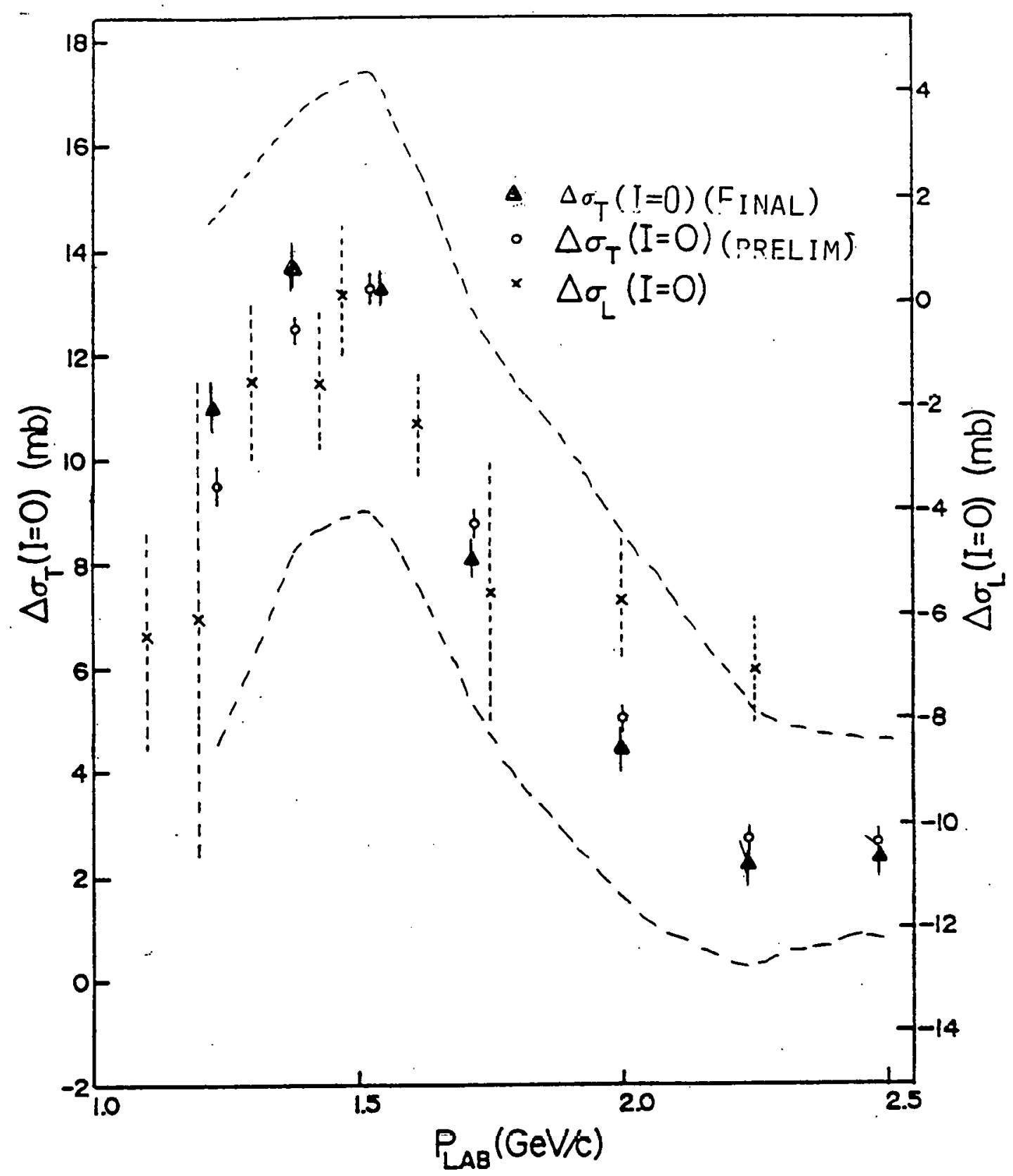

Figure 1

Plot of $\Delta \sigma_{T}(I=0)$ and the $\Delta \sigma_{L}(I=0)$ values shown on an offset scale to demonstrate the similarity of the structure in the two measurements. The absolute systematic error corridor is shown by the dashed curves for the $\Delta \sigma_{\mathrm{T}}$ measurement. 
2. Energy Dependence of the reaction $p \vec{p} \rightarrow p n \pi^{+}$and $p p \rightarrow d \pi^{+}$ Between 1.17 and $1.96 \mathrm{GeV} / \mathrm{C}[\mathrm{E}-462, \mathrm{ZGS}]$ [M.M. Calkin, M.D. Corcoran, G. S. Mutchler]

In the summer of 1979 the Rice Medium and High Energy Physics Groups, along with the Effective Mass spectrometer Group at Argonne National Laboratory carried out Experiment E-462. The ZGS Effective Mass Spectrometer was used to collect a high statistics sample of data of the reaction $p \cdot p \rightarrow \Delta^{++} n$ at incident momenta of $1.17,1.47,1.70$, and 1.96 GeV/c. The experiment obtained data with the incident beam polarized in both the transverse and longitudinal modes, and was designed to have acceptance over most of phase space. The purpose of the experiment was to determine if dibaryon resonance effects are present in elastic channels.

The data analysis was a multistep process which included track reconstruction from the spark chamber coordinates, reaction channel identification and separation, and efficiency determination of the various counters. After momentum-analyzed tracks were reconstructed from the spark chamber readouts; the restrictive kinematics of pp elastic and $\mathrm{d}^{+}$final states were identified and separated from the three body final states. The elastics and d ${ }^{+}$final states were used to calibrate the spectrometer, determine the beam 
polarization, and calculate observables to compare with previous experiments. The final set of programs separated pp $\pi^{\circ}$ from pn $\pi^{+}$final states with a zero constraint fit to the forward particles' momentum and direction of the recoil track. A maximum likelihood fit was used to extract a fit to the coefficients of the spherical harmonics $Y_{L M}$. The density matrix elements for the reaction $\rho_{n n}$ which are directly related to the spherical harmonics are then obtained.

At this point the data analysis is complete. Consistency checks of the results are being obtained by Monte Carlo simulation of the data and by an acceptance-free maximum likelihood calculation of the density matrix elements. The data are presented in Figures 2-4. Figure 2 shows the energy dependence of the polarization from 1.2 to $12 \mathrm{GeV} / \mathrm{c}$ (plotted vs. cose below $2 \mathrm{GeV} / \mathrm{c}$ and vs.jt for above $2 \mathrm{GeV} / \mathrm{c}] .^{2}$. Figures 3 and 4 show density matrix elements at $1.47 \mathrm{GeV} / \mathrm{c}$ for the spin-averaged and polarized cases respectively. This data analysis is part of the Ph:D. thesis of M. M. Calkin.

The triggering modes in this experiment collected $p p=d \pi^{+}$everits as well as thret body final states, and the analyzing power, $A_{y o}$ has been calculated for these events at 
all four energies. The large solid angle acceptance of the Effective Mass spectrometer allowed measurements of pion center-of-mass angles from $8-163^{\circ}$. The analysis of the pp-d $\pi^{+}$channel is complete, and a manuscript entitled "Analyzing Power in the Reaction pp $\rightarrow \pi^{+}$for Beam Momenta from $1.17 \mathrm{GeV} / \mathrm{c}$ to $1.96 \mathrm{GeV} / \mathrm{c}$," has been submitted to Physics Letters B [see copy attached to this report]. We find that $A_{y \circ}$ for this reaction shows very strong energy dependence in this region, as well as considerable structure in the angular distribution. None of the several recent theoretical calculations conly one of which includes dibaryons) adequately reproduces the data. This analysis was carried out by M.D. Corcoran with many helpful suggestions from A. B. Wicklund at Argonne.

2 The data above $2.0 \mathrm{GeV} / \mathrm{c}$ are from earlier. Effective Mass spectrometer experiments, reported by A. B. Wicklund

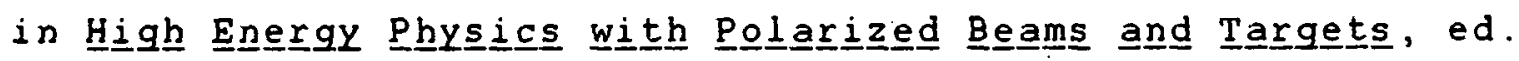
by M. I. Marshak (AIP, New York, 1976), p. 198 . 
Figure 2

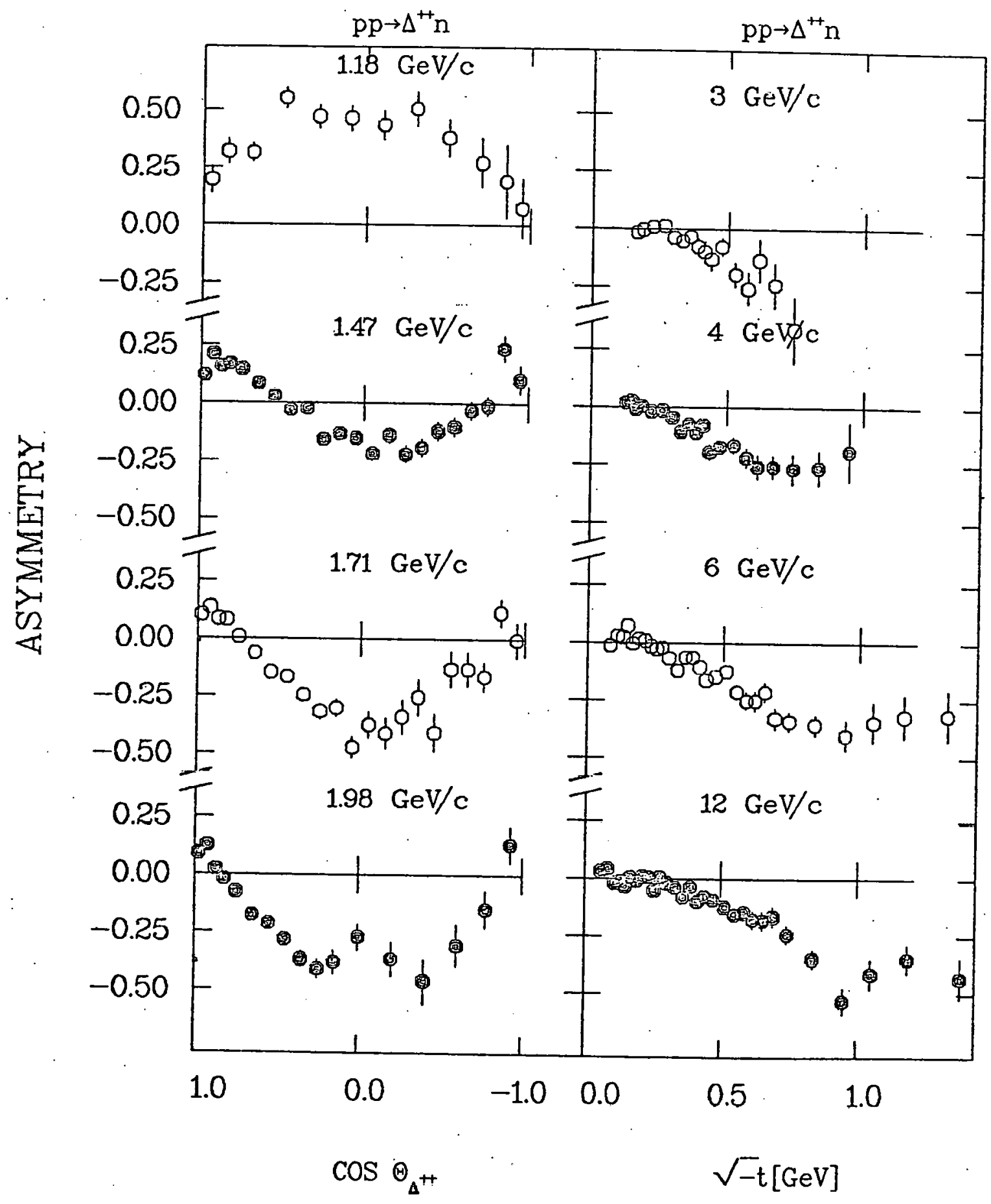


Figure 3

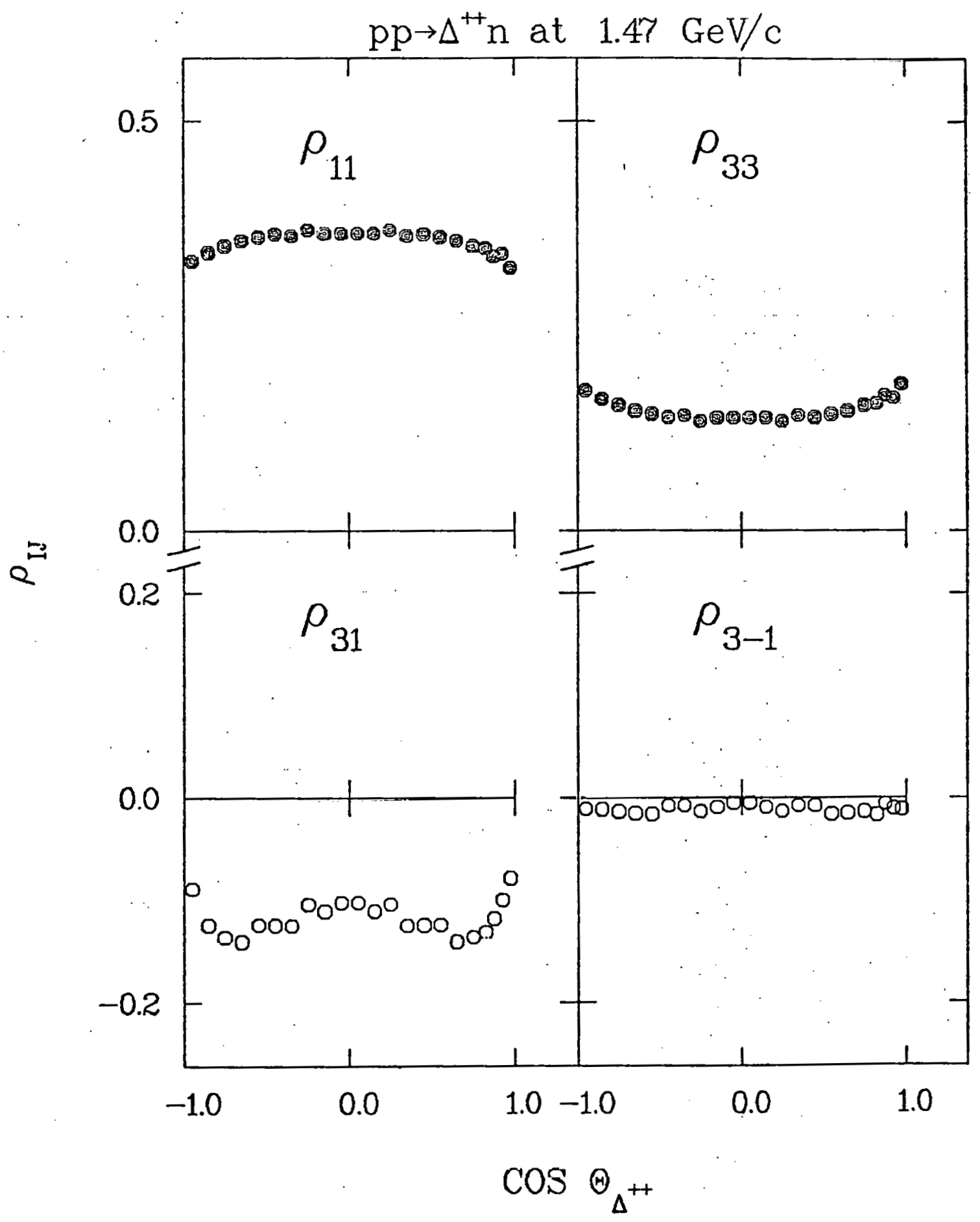


Figure 4

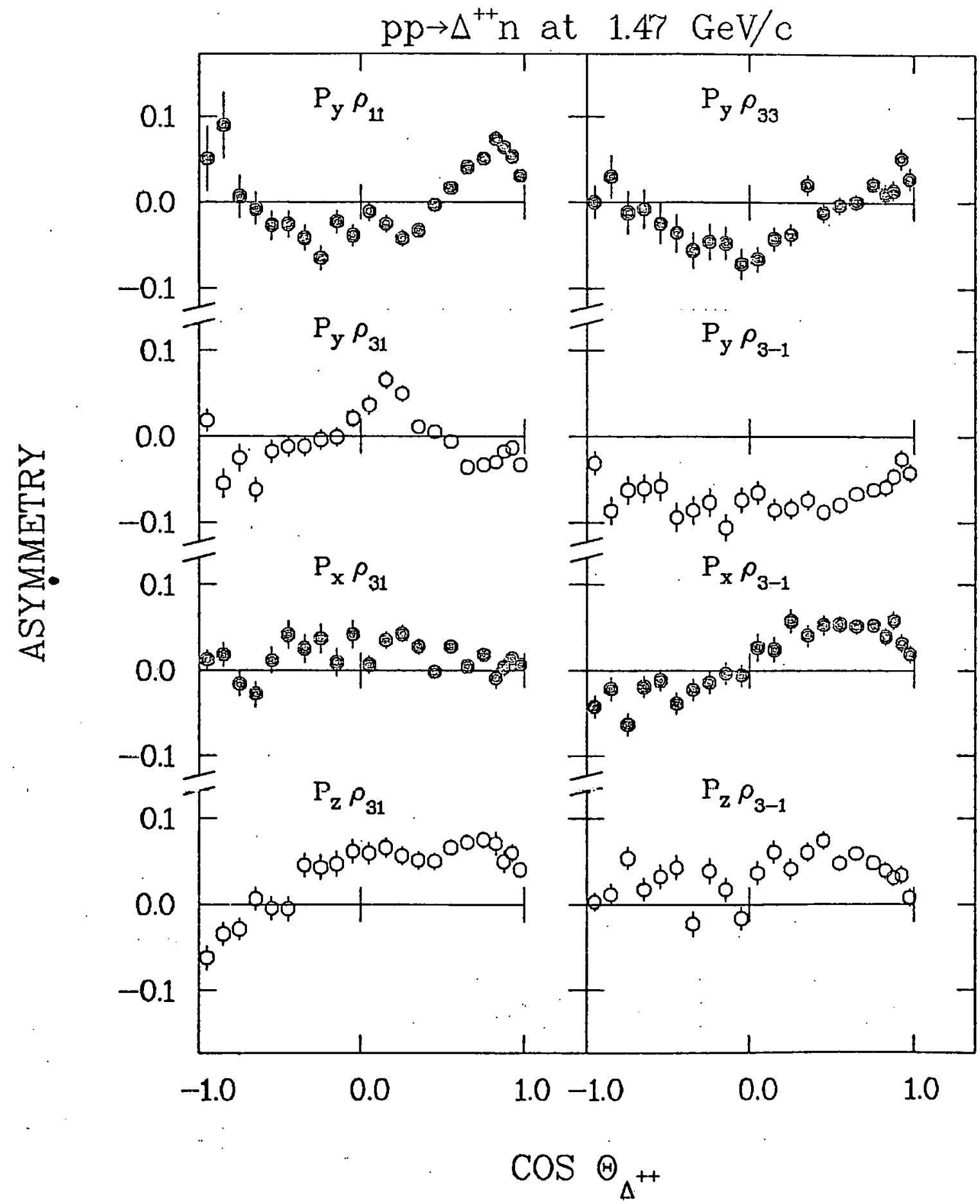


3. The Proton Beam Polarization Effort at BNL [J.A. Rice, J.B. Roberts, B.E. Bonner, G.C. Phillips, J.A. Buchanan ]

During the last year the Rice Group continued to participate in the collaboration that seeks to obtain useful polarized beams from the AGS at BNL. All the group meetings were attended and contributions were made to the overall system planning. In addition, James Buchanan of Rice served on the electronics/computer steering committee which arrived at the electronics system design and standards. The principal Rice contribution to this effort is assumption of the responsibility of design, construction, testing, calibration, and installation of the LINAC polarimeter, which is discussed below.

The main portion of construction of the polarimeter is complete. A stainless steel vacuum chamber with aluminized mylar windows for scattering portals exists. It has been vacuum tested and helium leak-checking has been completed. Polished scintillators and light pipes have been made and assembled with phototubes. The tubes have aluminum mounts which hold them in vertical and horizontal scattering planes. on the vacuum box. The target holder/changer is complete,but actual targets have yet to be fabricated. The 
analog and logic data from the phototubes will be analyzed via CAMAC inputs to an ISI-11 minicomputer. Electronic components are assembled except for wiring to the phototubes. Sensitivity of the polarimeter to beam position deviations has been calculated and will be taken into account on-line. Equations which maximize the accuracy of the polarimeter's measurements have been derived, and will be entered into the LSI-11 as software is developed. The polarimeter will be calibrated at The Indiana University Cyclotron Facility this winter and installed at Brookhaven. The design, construction, testing, installation, and description of this instrument will comprise the M.A. thesis of J.A. Rice.

4. The Polarized Proton Beam Effort at FNAL for E-704

The combined proposal P-704 to FNAL was approved during the November 1981 FNAL PAC meeting. Since then the FNAL POlarized Beam Collaboration has met on several occasions to discuss the division of responsibility for construction of the spectrometer for simultaneous measurement of several spin parameters in $p p$ and $\bar{p} p$ scattering at energies up to $200 \mathrm{GeV}$. The planned spectrometer is shown schematically in Fig.5. The magnetic spectrometer will detect single charged particles and $\Lambda^{\circ}$ 's produced at forward angles, and the lead glass calorimeter will detect $\pi^{\circ}$ 's and possibly direct pho- 
tons produced at $90^{\circ} \mathrm{C} . \mathrm{M}$. at high $\mathrm{p}_{t}$. We have agreed to work with the Annecy and Trieste Groups to develop polarimeters for the proton beam, to furnish the six $x-y$ proportional chambers P10-PIS and their associated electronics and readout system, and to build the threshold Cherenkov counters for the spectrometer. This apparatus is discussed in more detail in the Renewal Proposal. E-704 is presently shown as a first round Tevatron II experiment, and is expected to be installed by mid-1985. We have submitted a tentative experimental agreement to the Laboratory to be reviewed by its administration and by the Program Committee at the Fall 1982 Meeting. We anticipate that the agreement will be finalized by the end of the present contract year.

B. TET STUDIES

\author{
1. A Study of Structure of High p Proton-Proton Inter- \\ actions (Jets) [E-609, FNAI] [M.D. Corcoran, K.A. Johns, \\ H.E. Miettinen, J.B. Roberts, C.J. Naudet, J.A. Rice, \\ G.C. Ph1111ps]
}

The Rice High Energy Group in collaboration with Argonne, Fermilab, Lehigh, Penn, and Wisconsin has successful- 
ly completed the installation, calibration, and initial running of Fermilab experiment E-609. This experiment employs an 8 sr full azimuth segmented calorimeter to investigate the nature of high transverse momentum $\left(p_{t}\right)$ phenomena (jets).

In spite of severe problems with the cryogenic M6E beam line (which limited our running time) and poor beam structure, E-609 ran over $200 \mathrm{hrs}$ for tune-up and calibration purposes beginning in mid-February and completed 500 hrs of actual data taking from March until mid-May. Since then a diverse attack has begun on many phases of the data analysis including Monte Carlo studies, energy resolution studies, and tracking, as well as preliminary investigations of the data itself. In June, two preliminary reports were presented at the High Energy Conference in Paris. In addition, a Phys. Rev. Lett. is being written on these preliminary results. Some fraction of the data was taken with a variety of heavier nuclear targets. A Phys. Rev. Lett. on the A dependence of high $p_{t}$ cross sections is in preparation.

The motivation for E-609 follows from the parton picture of short range hadronic interactions. In this picture two incident constituents inside the hadrons undergo a hard scatter and then fragment, into particle jets with large 
transverse momentum. High yields of inclusive single particles at the ISR and results from small acceptance calorimeter triggers at Fermilab gave much support to these ideas.. However, trigger biases which allow events from non-hard scattering processes to simulate jets have made the interpretation of these experiments difficult. More recently, two groups, NA5 ${ }^{3}$ (Bari-Krakow-Liverpool-MPI-Munich-Nijmeger Collaboration) at CERN and $E 557^{4}$ (Fermilab-Illinois-IndianaMaryland-Rutgers Collaboration) at Fermilab, have measured high $p_{t}$ events using large acceptance full azimuth calorimeter triggers.

Their results show large cross sections, high multiplicities, and a lack of the simple planar structure that might be expected for jets. Hence the first questions $E-609$ wishes to answer are: 1 ) can one find unbiased triggers which cleanly select jet-like events [NA5 and E557 have found that only $5 \%$ of the events taken with full calorimeter trigger are possible jet candidates] 2) do these jet-like events reflect underlying jet structure or are they merely statistical fluctuations of the average non-planar events and 3) what mechanism is responsible for the majority of events which deposit large transverse momentum in the calorimeter only as a result of their large multiplicity. Other 
goals include measurement of

$$
E \frac{d^{3} \sigma}{d p^{3}} \rightarrow \text { jets }+x
$$

and extraction of the parton-parton cross section dô/d $\hat{t}$. We will also attempt to separate quark-quark and quark-gluon processes by means of charge and flavor identification.

\section{1:1: Apparatus}

The E-609 detector system is located in the M6E beam line at Fermilab. The experimental apparatus (Fig. 5) consists of a wall of muon veto counters, 12 planes of drift chambers, 3 planes of proportional chambers (built at Rice), a spectrometer magnet providing a small transverse momentum kick, and the segmented sampling calorimeter. In addition, some fraction of data was taken with an imaging Cerenkov counter which allowed simultaneous measurement of several secondary particle velocities. The calorimeter (Fig. 6) provides full azimuth coverage over $8 \mathrm{sr}$ at $400 \mathrm{GeV} / \mathrm{c}$. It consists of 528 individual modules stacked.together to form 132 longitudinal segments or towers. Modules in successive layers grow in cross sectional area so as to provide a telescoping tower structure which minimizes cascade shower 
cross- talk between adjacent segments. The "electromagnetic" front layer of the calorimeter consists of $5-8.5$ radiation lengths of lead/scintillator sandwich. The remaining three layers form the "hadronic " section of the calorimeter and consist of modules with iron/scintillator sampling giving a total of $20-24$ absorption lengths.

Track information for charged particles was provided by twelve planes of drift chambers and three planes of proportional wire chambers (PWC's). The PWC's and associated electronics were designed and constructed by J.A.Buchanan, M.D.Corcoran, and J.Windish at Rice and were installed in the experimental area and tested by K.A. Johns, C.J.Naudet, and J.B.Roberts. They were the first wire chambers after the hydrogen target and were necessary (rather than drift chambers) because of the high particle flux there. One plane measured the horizontal ( $x$ ) coordinate, and the other two had sense wires at \pm 15 degrees to the vertical to provide a measurement of both the horizontal and vertical ( $y$ ) coordinates and resolve ambiguities in matching $x$ and $y$ pairs. The active area of each plane was $63 \mathrm{~cm}$ (horizontal) by $33 \mathrm{~cm}$ (vertical), and the total number of instrumented wires was 1024 .

The drift chambers (constructed at Wisconsin) ranged in 
size from .9m (horizontal) by . .5m (vertical) to $2.4 \mathrm{~m}$ (horizontal) by $1.5 \mathrm{~m}$ (vertical), with a total of about 950 instrumented wires. Three of the drift chambers were "delay line" chambers; signals were taken from both top and bottom of the delay line to provide a measurement of both $x$ and $y$ coordinates.

1.2: Calibration of the calorimeter

Because of the steeply falling cross section of high $p_{t}$ events, the response of the calorimeter must be uniform everywhere. "Hot spots" or an extended tail on the $p_{t}$ pulse height spectrum can cause severe distortion of the measured cross section. To establish uniformity, mimimum ionizing muons were steered with a rotating dipole magnet through each of the 528 modules. Each module was balanced by adjusting the hv of its tube so as to give a response equal to the calculated energy deposited for straight through muons. Software for control of the rotating magnet and for some of the calibration data acquistion was written by K.A.Johns of Rice. In addition Rice has built counters for the calibration triggering system and has aided in the design of a remotely controlled movable frame system to transport those counters in the $x-y$ direction over the full area of the calorimeter. 
To establish the absolute energy scale, beams of hadrons and electrons at various momenta were steered into some subset of the calorimeter modules. Analysis of the energy resolution of the calorimeter is being carried out by. K.A.Johns as part of his M.A. thesis at Rice. Knowledge of the energy resolution is not enough to determine the absolute $p_{t}$ scale, however, for the steeply falling pt cross section greatly magnifies the difference between $p_{t}$ (true) and $p_{t}$ (measured). To establish the absolute $p_{t}$ scale, Monte Carlo studies including $\pi^{0}$ /hadron pulse height spectrum differences and energy sharing between adjacent segments must be done which convolute the energy resolution of the calorimeter with the $p_{t}$ cross sections of various triggers. This work will be pursued by K.A. Johns and M.D.Corcoran in the coming months.

\section{3: Tracking}

The study of quark-quark and quark-gluon differences depends crucially on the determination of charge, momentum and flavor of leading particles, all of which depend on tracking information from the wire chambers. Also, determination of the interaction vertex is necessary to remove spurious events and to correctly calculate the $P_{t}$. Since the charged multiplicity of a typical event is on the order of 
10-15, the track reconstruction is difficult and complex. A track reconstruction program is now operational on the VAX at the Physical Sciences Laboratory at the University of Wisconsin and will be brought to Rice now that our VAX is installed. R.Hollenhorst, C.Kuehn, A.Hasan (from Wisconsin) and M.D.Corcoran (from Rice) are involved in the track reconstruction. Presently the program finds tracks with about $90 \%$ efficiency, and we expect additional improvements in that number. A hardware tracking module has been constructed at wisconsin and is nearly complete. It should greatly improve the speed of track reconstruction.

1.4: Triggering

E-609 employed a powerful triggering system which allowed data to be taken with simultaneous use of approximately 200 different trigger conditions. Most of these triggers were geometrical and required the summed transverse energy $\left(E_{t}\right)$ in particular regions of the calorimeter to be greater than some threshold $E_{t}$. These regions could be as small as one segment $(0.6 \mathrm{sr})$ or as large as the entire calorimeter ( $8 \mathrm{~s} r)$. A fraction of the triggers were used to study phenomena such as non-coplanar jets and to search for three jet events. Preliminary analyses of two of the triggers, a global trigger and the "two high" trigger, have been presented 
at Paris 5 and a reprint is attached [see "Publications"]. A brief summary of those results is discussed below.

1.5: Preliminary results

One of the more promising triggers we have investigated is the "two high" trigger which required solely that any two or more segments in the calorimeter each yield a $p_{t}$ greater than $1 \mathrm{GeV} / \mathrm{c}$. Such a threshold is high enough so as to preclude the event from being generated by low $p_{t}$ "bubble chamber" single particle distributions yet low enough so as to include hadrons which have fragmented from partons having transverse energy of a few GeV/c. Note both the "two high" trigger and the global (full calorimeter minus the outermost wings) trigger are apparently free from any geometrical bias. The global planarity distribution (Fig. 7) is similar to that observed by NA5 and $E 557$ and shows the majority of events to be noncoplaner (roughly only 5z-10z of the events have planarity greater than 0.8). (Note planarity $P$ is defined by

$$
\frac{\Sigma_{\max }-\Sigma_{\min }}{\Sigma_{\max }+\Sigma_{\min }}
$$

where $\Sigma_{\max }$ and $\Sigma_{\min }$ maximize and minimize, respectively the 
sum of $p_{t}^{2}$ in the $p_{t} x$ and $p_{t} y$ planes.) By contrast, the planarity distribution of the "two high" trigger (Fig. 8) though shows these events to be decidedly more planar (and we emphasize again that the "two high" trigger requires only that only two or more segments each give a signal larger than $1.0 \mathrm{Gev} / \mathrm{c}$ transverse momentum). Both distributions used events for which the total Et was greater than 11 Gev/c.

In partial answer to the question of whether those events which possess a high degree of planarity are the result of some hard scattering process or simply statistical fluctuations of non-correlated particles, the azimuthal angles of the "hadrons" from the events used in the distributions were randomized while maintaining the observed degree of momentum unbalance on an event by event basis. (Here we have defined "hadrons" via a simple clustering algorithm which grouped the highest $p_{t}$ segment with its highest $p_{t}$ neighbor. These two segments were then removed from the list of available segments and the process continued until all segments with $\mathrm{p}_{\mathrm{t}}$ greater than $0.3 \mathrm{Gev} / \mathrm{c}$ are included.) The resulting planarity distributions are shown as dashed lines in figs. 7 and 8 . From this we tenatively conclude that approximately half of the "two high" triggered 
events with planarity greater than 0.8 are possible jet candidates and cannot be accounted for by fluctuations of those non-planar events which characterize the global trigger.

A high degree of planarity is not sufficient by itself to determine whether an event shows simple di-jet character or not. In addition one must observe: (1) clustering of high $p_{t}$ segments in small enough solid angle so as to be distinguishable above the low $p_{t}$ background, (2) coplanarity in the azimuthal angle and, (3) approximate $p_{t}$ balance between the two jets. The degree to which many of the "two high" triggers satisfy the above conditions is exemplified by the three dimensional representation of a quite typical "two high" event shown in Fig. 9. The heights of the peaks which rise out of the front view of the calorimeter are proportional to the $p_{t}$ deposited in the calorimeter segments. To give an approximate scale, the height of the highest peaks correspond to $p_{t}$ 's of 1 - 2 Gev/c. The clustering of "particles", many with individually high $p_{t}$ 's, contrasts sharply to the total absence of signals everywhere else in the calorimeter (signals as small as $0.025 \mathrm{GeV} / \mathrm{c} \mathrm{p}_{\mathrm{t}}$ will appear in this plot). For comparison; a typical global event is presented in Fig.10. Typical global events satisfy the 
$E_{T}$ trigger merely by the large multiplicity of small $\mathrm{P}_{\mathrm{T}}$ fragments and show no irregular correlations betwen particles.

By making an additional cut requiring the number of segments with $0.5 \mathrm{Gev} / \mathrm{c}$ or more to be greater than 5; more than half of those. "two high" triggered events with Et greater than 11 show clear di-jet structure. Fig. 11 plots the $p_{t}$ distribution vs in $10^{\circ}$ bins for the first six events which satisfied the above criteria. Clustering and a high degree of coplanarity are clearly seen in events 2,3 , 4, and 6. Again note there is relatively little transverse momentum deposited outside the two clusters.

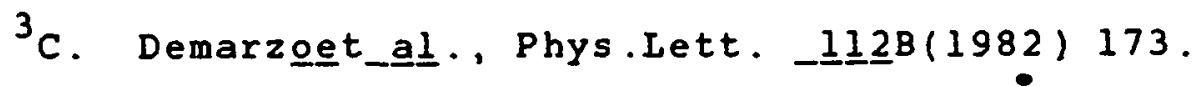

${ }^{4}$ B. Brown_et_al . Phys.Rev.Lett. _49 (1982) 711.

${ }^{5}$ M.Arenton_et_al . "Evidence for Jets from a Transverse Energy Triggered Calorimeter Experiment at Fermilab," submitted to XII High Energy Conference, Paris, July, 1982. 
Figure 5

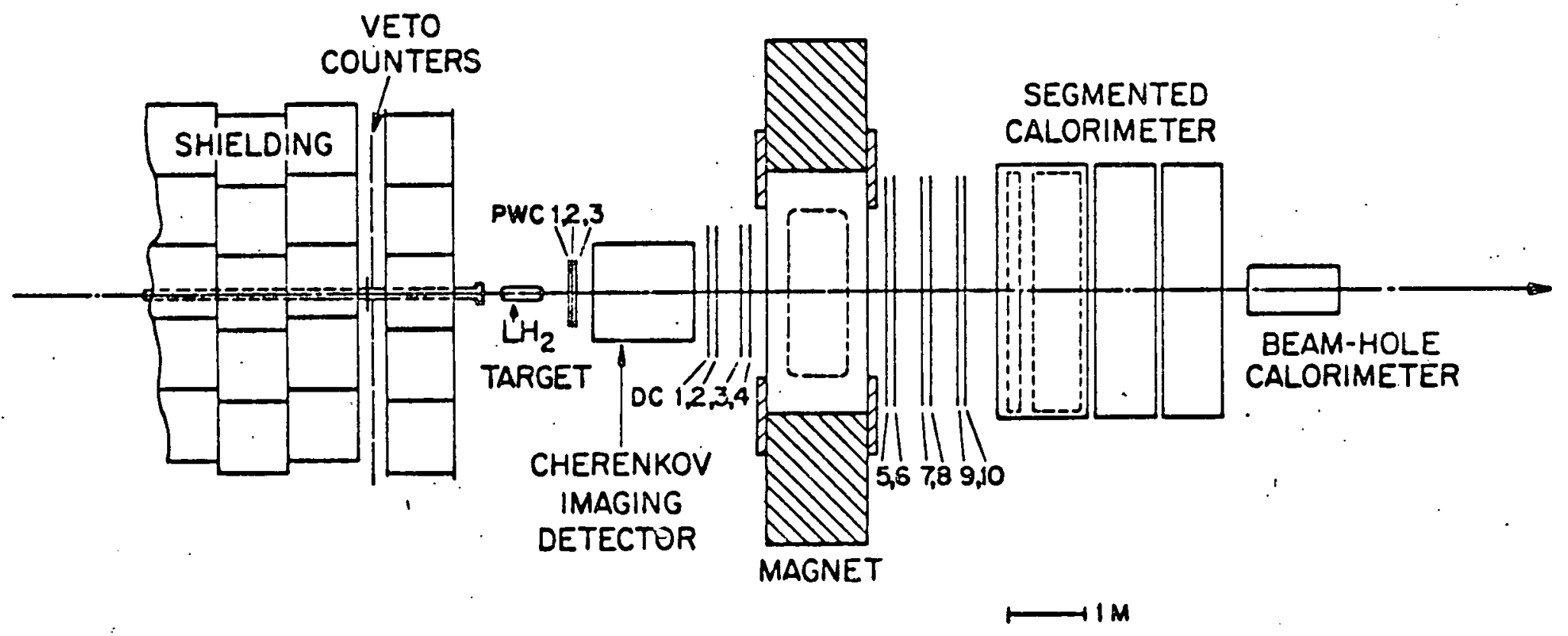

TOP VIEW OF E-609 APPARATUS 
Figure 6

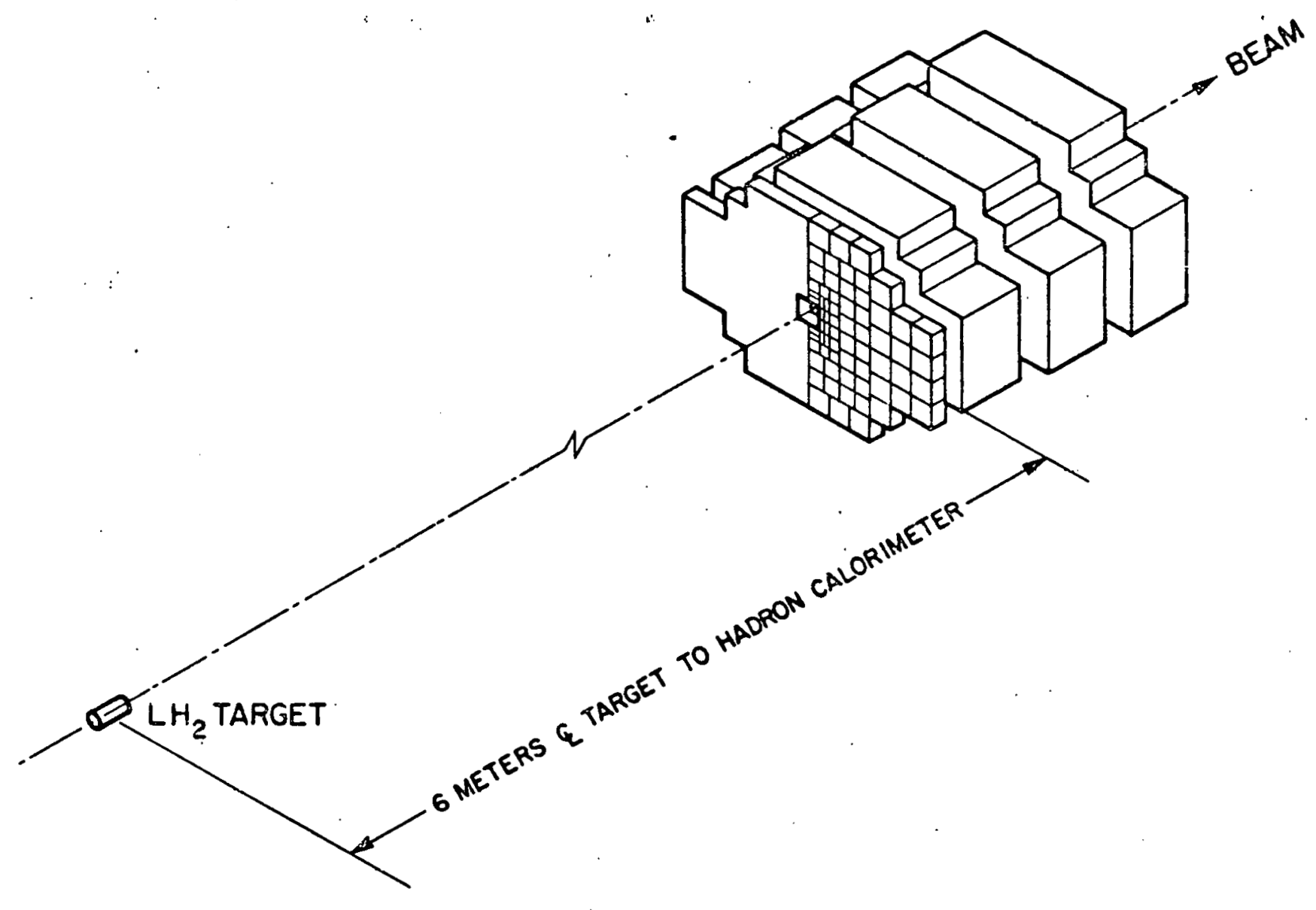

SEGMENTED REPRESENTATION OF CALORIMETER ARRAY 
Figure 7

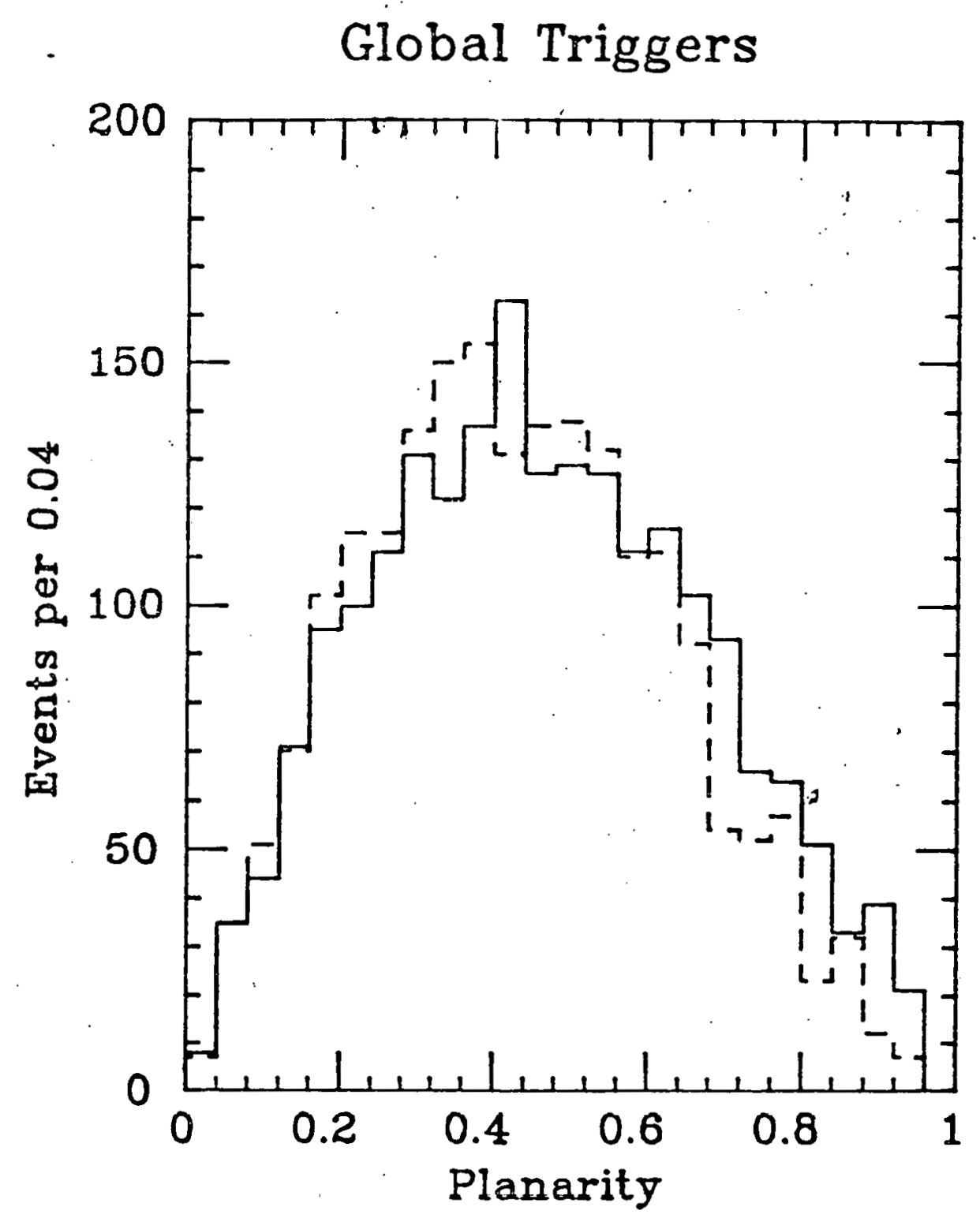


Figure 8

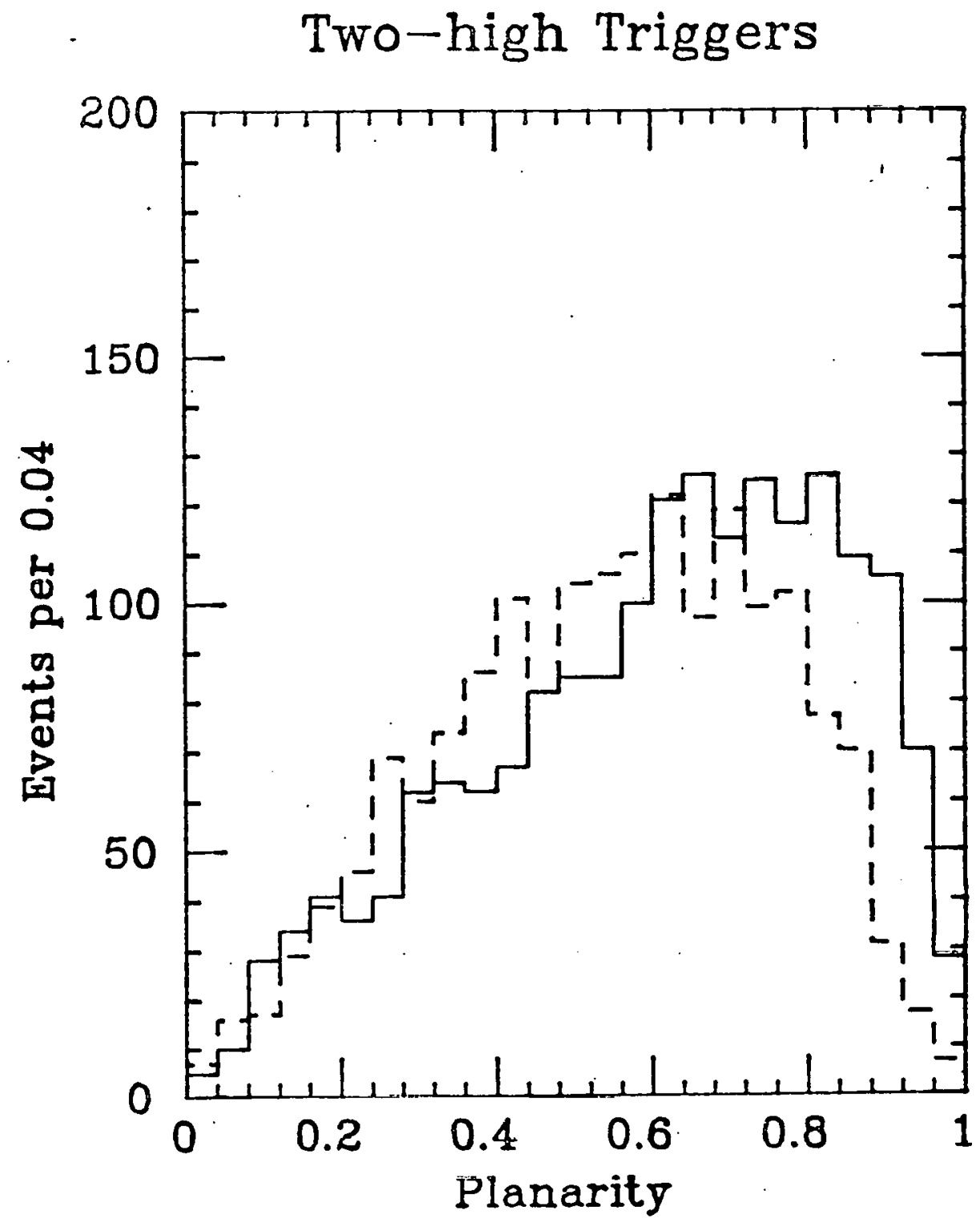


Figure 9

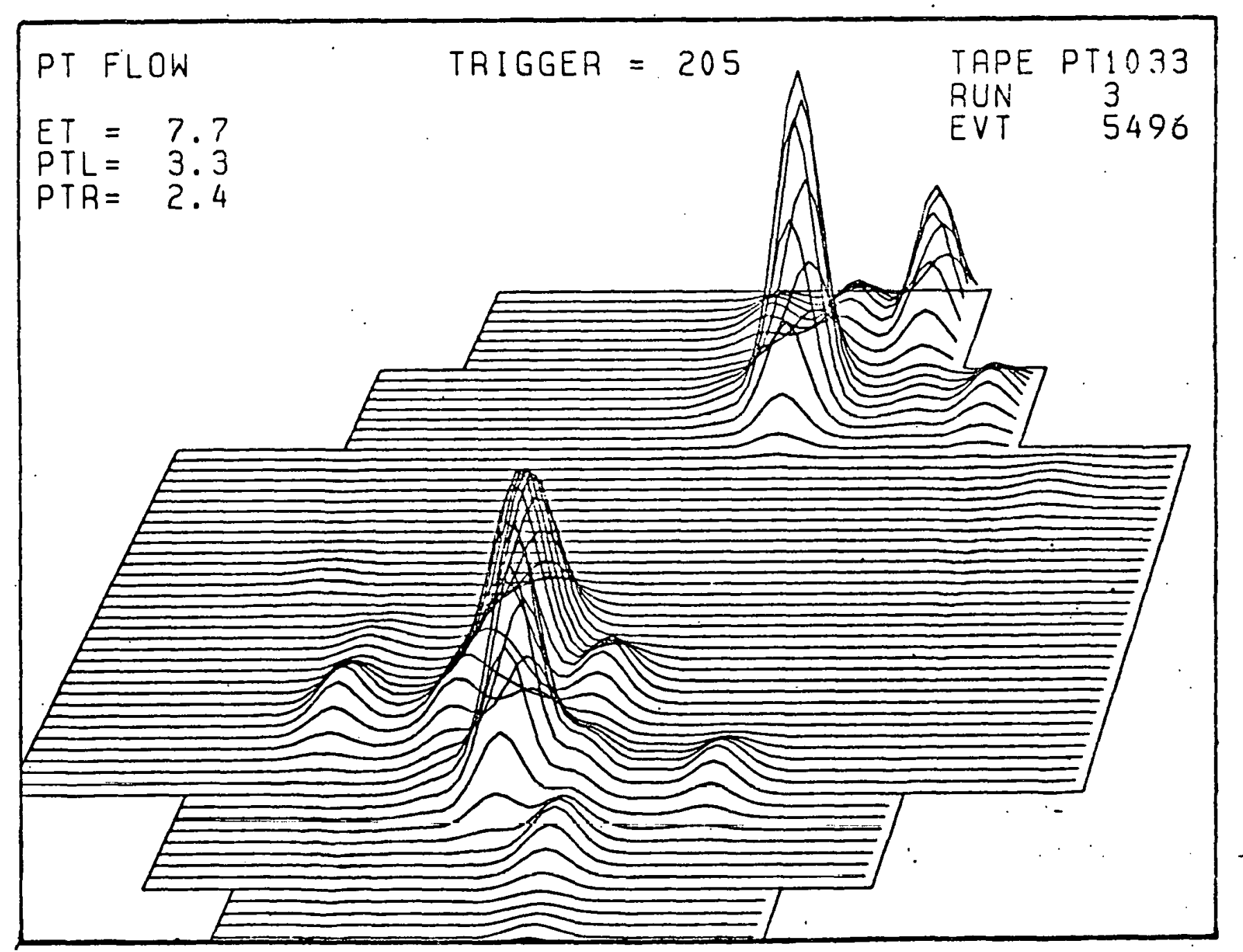


Figure 10




Figure 11
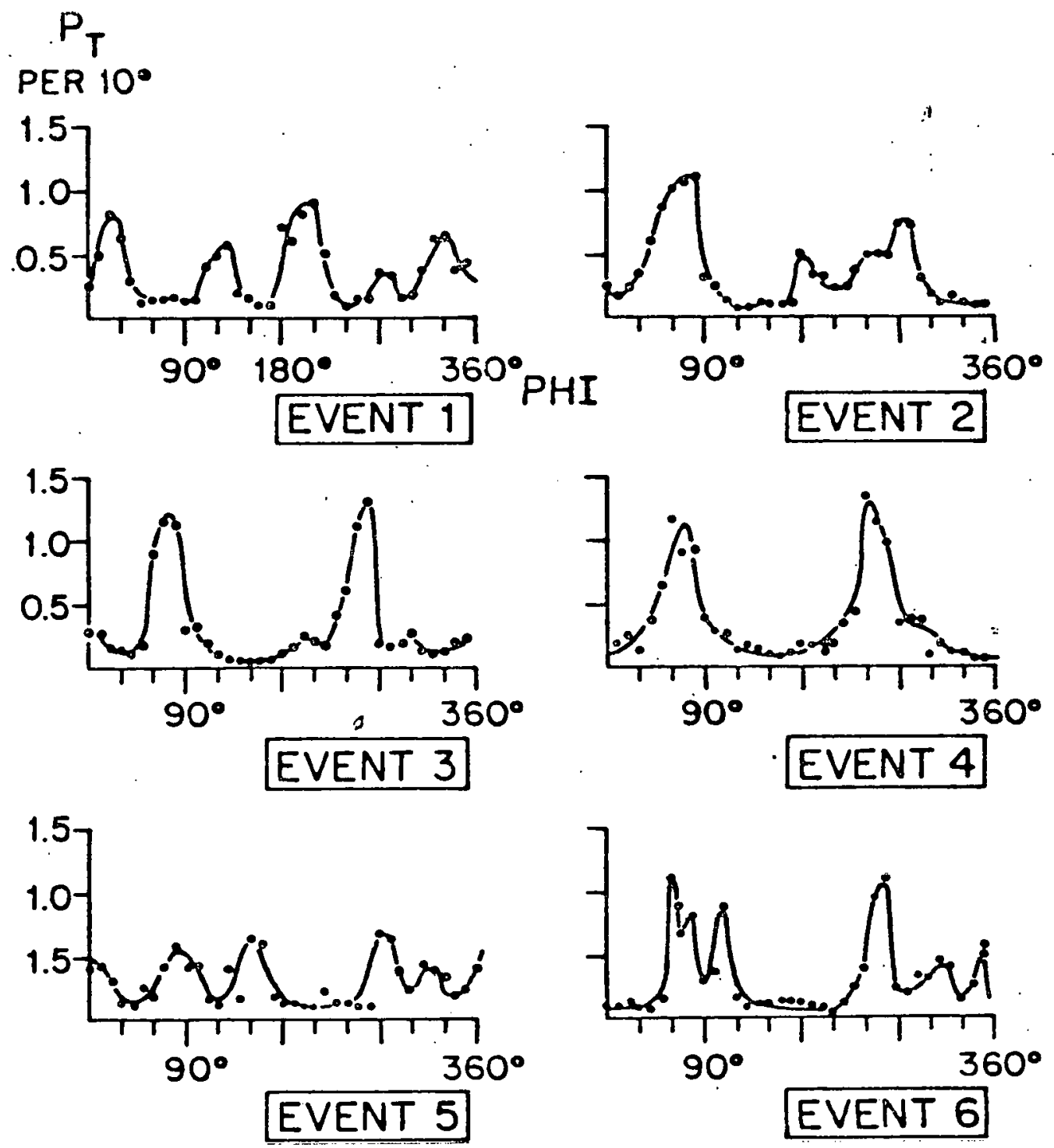
2. Monte Carlo Simulations of Jet Phenomena [H.E: Miettinen, M.D. Corcoran, C.J. Naudet, K.A. Johns ]

During the year we have continued our extensive Monte Carlo studies related to E-609. Detailed Monte Carlo calculations in jet physics have become a necessity in view of the fact that the theory (QCD) only predicts part of what it takes to calculate experimental observables. The interpretation of the results from CERN NA-5 and other experiments remains somewhat controversial; one topic of particular interest is the role of parton bremsstrahlung in high transverse energy events. To study these and other questions, we have obtained and implemented on a VAX $11 / 780$ at Rice a "state of the art" high $P_{T}$ Monte Carlo, written by G. Fox and collaborators at CalTech. ${ }^{6}$ This programblends perturbative $Q C D$ and phenomenological fragmentation to model hadronic final states in $\mathrm{e}^{+} \mathrm{e}^{-}$annihilation and high $\mathrm{p}_{\mathrm{T}}$ hadron collisions, and has been very successful in explaining some of the NA-5 results. We are currently investigating the sensitivity of the results of this model to the phenomenological input. In the coming year we plan to make very detailed comparisons of this model to E-609 data under different triggering conditions in order to learn about lie underlying theory. This work is being carried out by 
9.E.Miettinen and Kenneth Johns, and will constitute a large part of Johns' Ph.D. thesis.

The "standard" (at least until recently) QCD Monte Carlo is that of Field and Feymman ${ }^{7}$, which uses first order QCD cross sections and a well-known fragmentation procedure to generate high $p_{t}$ jet events. Since this model does not include gluon bremsstrablung in either the initial or final states, a comparison of this model with that of Fox should help clarify the role of gluon bremsstrahlung in high $p_{t}$ events. The Field-Feynmann Monte Carlo is presently running on the Bonner Labs' PDP $11 / 45$ and will be transferred to the VAX as soon as possible. We are currently studying the effects of the input parameters and the structure functions on the generated events. As with the Fox Monte Carlo, we will make a comparison of the Monte Carlo generated events with the E-609 data. Implementation of the Field-Feynmann Monte Carlo is being done by M.D.Corcoran. A senior student, Gordon Carrie, assisted during the last year.

The work on a cylindrical phase space Monte Carlo [described in last year's report] was completed during the year. This study was Charles Naudet's M.A. thesis; he is currently in the process of finishing his report and will 
defend his thesis in October 1982. The results of the study will be used extensively in the analysis of E-609 data.

We are also working on developing a jet-finding algorithm to be used in the analysis of E-609. The algorithm is based on a "Gaussian smearing" method, originally developed by a group at the CERN ISR. ${ }^{8}$. The method will be tested and the parameters optimized using $e^{+} e^{-}$jets, and it will then be applied to E-609 data. This work is being carried out by Robert Norsworthy as his Senior Research Project under the supervision of H.E. Miettinen.

${ }^{6}$ C. Fox, Lectures at the 1981 SLAC summer school, CALT-68-863 (1981); G. Fox and R. Kelly, CALT-68-890 $(1982)$.

7 R.D.Field and R.P. Feynman, Nucl.Phys.B136 (1978) 1. 8 M. Albrow_ett_al_., Nucl.Phys. _B彑 16 o (1979) 1.

3.High Energy p-Nucleus Collisions [E-609, FNAL] [ J.A.Rice J.B. Roberts]

Proton-nuclear collisions are studied because they may yield information about the passage of partons through nuclear matter. If di-jet interpretations are true, and partons are stripped from incident protons, then the parton cannot hadronize for a distance of the order of 


$$
L={ }_{8}^{8} g_{1 a b} \Delta t \times c={ }_{1 a b} \times r_{\text {hadron }} \approx 80 \text { Fermis }
$$

where ${ }^{1}{ }_{1 a b}$ is a Lorentz factor and $r_{\text {hadron }}$ is a typical hadron radius. The largest nuclear diameters are of order 15 Fermis, so the parton flies through the nucleus before hadronizing.

In May, eight beam hours of data on $400 \mathrm{GeV}$ proton-nuclear collisions were obtained. This experiement was initiated and carried out by J.B.Roberts and J.A.Rice and will form part of the latter's Ph.D. thesis at Rice. The experiment consisted of placing nuclear target foils between the liquid hydrogen target and the PWC, shown in Fig.12, in relation to the detection system. The runs consisted of $H, C, A l, C u, S n$, and $\mathrm{Pb}$ targets plus an empty target run to determine background event rates. The calorimeter triggers used include global, double arm, and single arm, which key on events depositing transverse energies in excess of trigger thresholds in regions of $8 \mathrm{sr}, 4 \mathrm{sr}$, and 2 sr solid angle ( $\left.\Omega^{\star} p p\right)$ in the calorimeter The additional triggers employed are the "two-high" $E_{T}$ triggers described in the preceeding section, and a low multiplicity global trigger, which operates like the global except that it additionally constrains particle multiplicities to be below a 
set threshold.

In Fig.12 we see that the usual cross-section scaling of

$$
\sigma\left(E_{T}\right)=\sigma_{0} A^{\propto\left(E_{T}\right)}
$$

holds for global, double arm, and single arm data for $A_{ \pm 12}$, where $\sigma_{0}$ is constant, $A$ is target atomic number, and $\propto$ is a parameter that is dependent upon $E_{T}$, However, $H$ falls below the $A^{\alpha}$ extrapolations in each case, with the deviation growing as the trigger solid angle decreases. In Fig.13, we see that $A^{\alpha}$ scaling holds for $A_{ \pm} 2$ for the "two-high" $E_{T}$ and low multiplicity global triggers and that $H$ falls above extrapolations. When the "two-high" $E_{T}$ trigger is cut in software to yield only events whose two highest $E_{T}$ segments sum to greater than $3 \mathrm{GeV}$ of $E_{T}, A^{\alpha}$ scaling completely breaks down. All curves but the global trigger in Fig.12 represent never-before seen trends. 


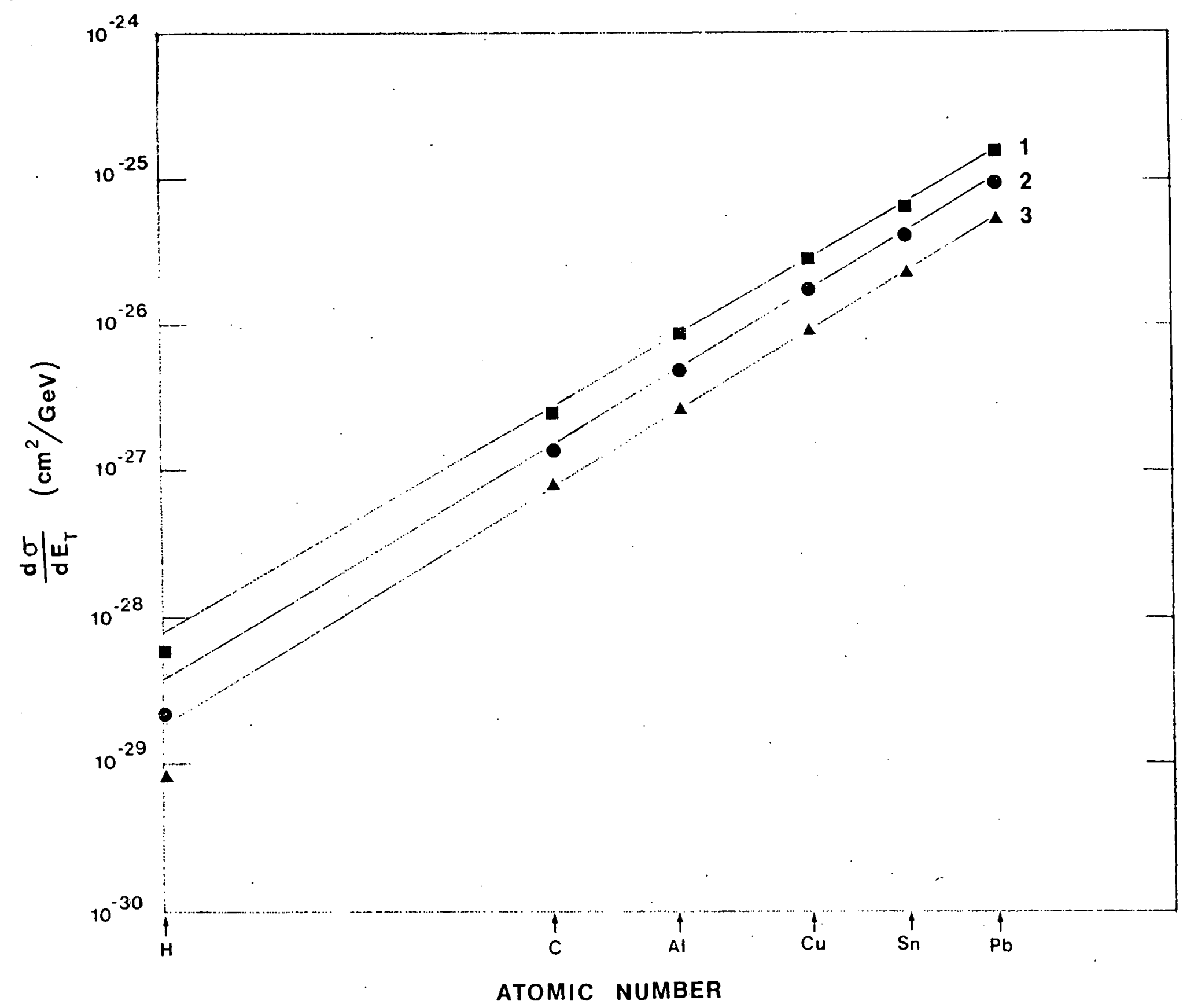

Figure 12:The A dependence of cross-sections summed ovel.

the Erof the whole calorimeter for an $E_{T}$ range of

9 ihrough $12 \mathrm{GeV}$; ior global (1), double arm (2), and single

arr ( 3 ), triggers. Lines it $A \geq 12$ points. 


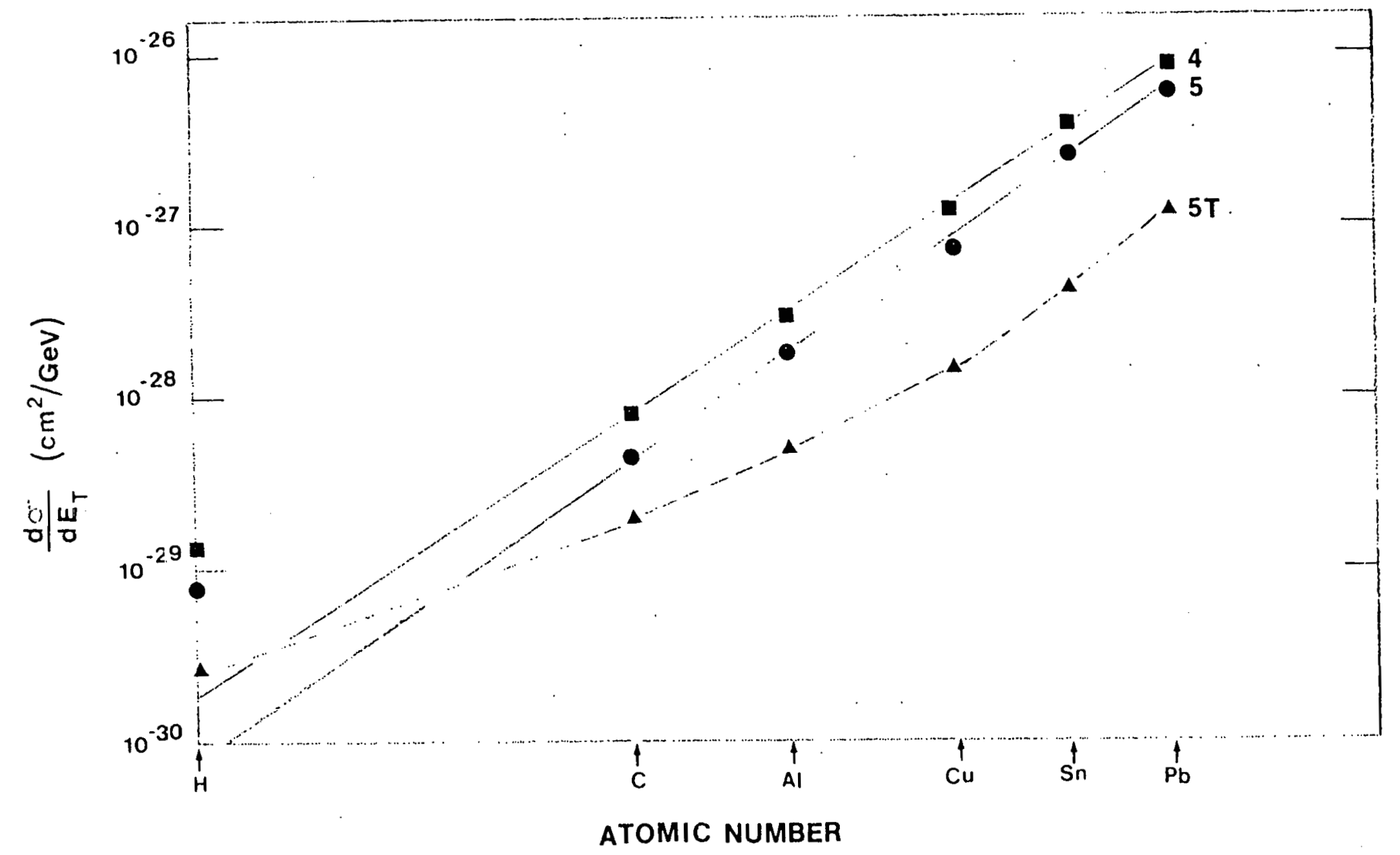

Ficure 13: The A dependence for cross-sections with $\mathrm{E}_{T}$ sumncd over the irhole calorimeter in a range of 9 through $12 \mathrm{GeV}$ tor low multiplicity global (4), and two-high E (5) triggers;

and for E.r in the range of 3 through $5 \mathrm{GeV}$ in the sum of the

two highest culorimeter segments for the two-higln-E trigrer (5T)

Solid liues it $A \geqslant 12$ data, while the daslued line guides the eye. 
C. OTHER PROJECTS

During the year effort was expended in pursuing various other topics viewed to be at the forefront of high energy research. These are discussed below.

1. Neutrino Oscillations [G.C.Phillips, G.S.Mutchler, J.B.Roberts, E.A.Umland, M.Duong-Van]

The proposal (P-559, LAMPF) to search for "appearance oscillations," of neutrinos was rejected by the LAMPF PAC. Since we believed that our proposed technology was uniquely advantageous for the detection of the oscillation of $v_{\mu}$ to $v_{e}$ by the reaction:

$$
v_{e} e^{p+e} n
$$

with coincident detection of the $e^{+}$and the (time delayed) neutron, we proposed it as an instrumentation development project during the year. No official response has been received as of this date; however, we believe it is, likely that this proposal may be rejected. Nevertheless, we continue our interest in this exciting field.

2. High Energy e-p Collisions [J.B.Roberts, G.C.Philli ps ]

The lack of progress on possibly building an e-p col- 
lider at FNAL or ISABELLE has been a disappointment; however, we continue to be very interested in this area of physics.

3. Underground Physics [G.S Mutchler, J.B.Roberts, G.C.Phillips]

The proposal to develop a national underground facility for particle and nuclear research is of great interest to our group, and we have attended meetings and contributed to this on-going effort.

4. Super-LAMPF [B.E.Bonner, J.B.Roberts, G.C.Phillips]

The interest in using the intense LAMPF beams by acceleration to higher energies to allow new areas of research (e.g., Kaon and anti-nucleon beams) is of interest to our group and during the year we participated in two meetings at LANL on this subject.

D. INSTRUMENTATION

\section{Proportional Wire Counters [J.A.Buchanan]}

Two 30.4 in $\times 11.2 \mathrm{in}$. PWC's were modified to accept one mil diameter sense wires and our new E-609 type 64-wire amplifier- discriminator units. It was necessary to generate new artwork and PC boards for the sense-wire plane inserts. Considerable machine work by the University shop was required to complete the adaptation. 


\section{PWC Electronics [J.A.Buchanan]}

Fourteen) new 64-wire amplifier-discriminator units were built. These units were interfaced to our existing multi-coordinate readout system so they could be integrated easily into our data acquisition system. With this added capacity, we can now instrument 2112 wires with these East-amplifier modules.

3.Polarization Gate Module [J.A.Buchanan]

A new CAMAC Pol Gate module has been designed and built to derive appropriate control signals from the polarimeter signals supplied from LAMPF CCR. These supplied signals are: Normal or Reversed polarization and Polarized or quenched mode. The new module causes all events to tape to be tagged with bits indicating $N, R, P$, and $Q$. The polarimeter outputs, ion chamber and time are scaled separately for both $P$ and $Q$. To save hardware scalers, the scalers are read and cleared at the transitions $N-R$ or $P-Q$ and accumulated into separate software locations. The Q-P data may then be used to measure the beam polarization (independently from the polarimeter). Quenched data may also be used to measure instrumental asymmetries in the polarimeter and in our apparatus (see IASL Memo MP-13/MWM/I80-10). 


\section{Computer Progress [J.M.Clement]}

The changeover to RSX-11M for data acquisition was tested this summer during Experiment E-336 at LAMPF, and it was a success. The CAMAC language compiler simplified the process of specifying data acquisition parameters. Hopefully the CAMAC language can be extended and further developed.

5. The VAX-11/750 [J.M.Clement and J.A.Buchanan]

The VAX-11/750, purchased by Rice University, is currently being installed in the Bonner Labs.Plans for the future use of it include translating all of the PDP-11/34 data analysis code to VAX $11 / 750$ native code. This will allow replay of data tapes quickly on the 750 without using the slow PDP-11 emulation mode. Future plans include acquisition and installation of terminals in various users ' of fices. The ready availability of a terminal near the user's desk is vital to efficient computer use. This keeps the computer resources near the user's notes and reference material. An immediate idea can be implemented while it is still fresh. Remote facilities tend to be a barrier to good us age .

The acquisition of a new Florida Data printer has given us added print capabilities. The Florida Data printer, in 
addition to graphics and normal computer printouts, is capable of publication-quality printing. RUNOFF, a program available free from the DECUS, has been extensively modified to support the Florida Dataprinter. With this new facility, publication of results should be speeded up. Typographical errors in the final paper should be reduced as well.

E. THEORY [Ian Duck and Eric Umland]

The cloudy bag model of Thomas and DeTar 9,10 has been used to calculate the masses and pionic couplings to nucleon and delta channels of nucleon resonances. The cloudy bag model restores chiral invariance to the MIT quark Bag Model Lagrangian by invoking a massless spin zero Goldstone boson taken to be the pion. An immediate consequence is a pionquark coupling term in the static spherical bag, weak pion field limit:

$$
\operatorname{Lim}=\frac{1}{2 \mathrm{f}} \int \mathrm{d}^{3} s \frac{\phi}{q(\mathrm{x})} \quad \gamma_{5} \vec{\tau} \mathrm{q}(\mathrm{x}) \cdot \vec{\phi}(\mathrm{x}) \delta(\mathrm{x}-4)
$$

where $q$ and $\dot{\phi}$ are bagged quark and free pion wavefunctions, $f$ is the pion decay constant and the delta function constra- 
ins the interaction to the bag surface at radius $R$. This interaction has been successfully used to calculate pionic corrections to the static properties such as magnetic moments, masses, and axial coupling constants of the lowest lying baryon multiplet. Ve have extended these calculations to radially excited states of the nucleon, in particular the $P_{11}(1470)$ nucleon resonance, the $N^{*}$. The $N^{*}$ is considered to be a $(2 s)(1 s)^{2}$ three quark configuration. It is a doublet of internal $s u(6) \times 0(3)$ symmetry states with one a member of 56 and the other a member of a 70 multiplet. They are degenerate if one neglects gluonic and pionic effects. We find their lowest order mass in the MIT bag model to be $1.55 \mathrm{GeV}$ after correcting for CM motion of the quarks about the static bag origin. Pionic and gluonic radiative corrections (see Figs.14 and 15) mix the two states, yielding physical masses

$$
\begin{aligned}
& M_{A}=1.393 \mathrm{GeV} \\
& M_{B}=1.511 \mathrm{GeV}
\end{aligned}
$$

These are in remarkably close agreement with the two level $\pi N$ phase shift analysis of Ayed, who claims

$$
\begin{array}{lll}
e+p & M_{A}=1.413 \mathrm{GeV} & (3 \mathrm{a}) \\
e+p & M_{B}=1.532 \mathrm{GeV} & (3 \mathrm{~b}) .
\end{array}
$$

We have also calculated $N * N \pi$ and $N * D \pi$ coupling con- 
stants [see Fig. 16] and partial widths including pionic vertex corrections [Fig.17]. We find

$$
\begin{array}{ll}
r\left(N_{A}^{*} \rightarrow N \pi\right)=91 \mathrm{MeV} & (4 \mathrm{a}) \\
\Gamma\left(N_{B}{ }^{*} \rightarrow N \pi\right)=33 \mathrm{MeV} & (4 \mathrm{~b}) \\
\Sigma \Gamma\left(N_{i}{ }^{*} \rightarrow D \pi\right)=77 \mathrm{MeV} & (4 \mathrm{c}) .
\end{array}
$$

to be compared with Ayed's 11

$$
\begin{aligned}
& \Gamma_{A N \pi}=98 \mathrm{MeV} \\
& \Gamma_{B N \pi}=12 \mathrm{MeV}
\end{aligned}
$$

and Particle Group Data compilation: 11

$\Sigma \Gamma_{N \star D \pi} \sim 50 \mathrm{MeV}$. Again the agreement is exceilent considering uncertainties in the data.

At present we are calculating properties of "gluonic nucleons" (Ng) which have a 3 quark \{su(3) color dimensionality:8\}-1 gluon configuration in an overall color singlet $(8 \times 8-1)$. There are two $I=1 / 2, J=1 / 2, N g$ states: one whose quarks are in a $S_{\text {TOT }}=3 / 2$ state and another with $S_{\text {TOT }}=1 / 2$. They are degenerate until \{SU(6)\} symmetry is broken. Preliminary results indicate a mass for the lower state of around $1.67 \mathrm{GeV}$ and a partial width into $\pi N \sim 30 \mathrm{MeV}$. This is narrow enough to have been missed in elastic $\pi N$ scattering experiments in this region. The higher mass state is essentially decoupled from the $\pi N$ channel. This result suggests that new experiments may be needed. 
We have also identified an interesting discrepancy between theory and experiment for the value of the $\Delta \Delta \pi$ coupling constant. Arndt et al ${ }^{2} 12$ deduce $f_{\Delta \Delta \pi}$ from an analysis of $\Delta$ production in $\pi^{-} p \rightarrow \pi^{+} \pi^{-} n$ reactions, obtaining $f^{\text {Arndt }}{ }_{\Delta \Delta \pi}=.46+.11$. Our cloudy bag calculations give ${ } \Delta \Delta \pi$ )theory -.74 . This calculation is in good agreement with one using a very different approach ${ }^{13}$. We believe more experimental input is needed here.

Finally, we have worked on the soliton bag model of Friedberg and Lee 14 which employs a scalar field in a quartic polynomial in the Lagranian. The non-linear nature of the resulting equations of motion causes quark confinement in a dynamical fashion. In collaboration with Rudy Goldflam of the University of Washington, we have shown how the addition of a fundamental pion field renders the soliton-quark coupling term, go $\vec{q} q$, chirally invariant, leads to a Goldberger- Treiman relation, and reproduces the cloudy bag calculation of $f_{\pi g q}$.

We will submit four papers on these topics: on $f_{\Delta \Delta \pi}$, reference 15 , on the $N^{*}$, reference 16 , on the $N^{G}$, reference 17, and on the chirally invariant soliton theory, reference 18. Eric Umland will defend his Ph.D. thesis on these topics in October 1982 . 
${ }^{9}$ S. Thiberge, A. W. Thomas, G.A. Miller, Phys.Rev.

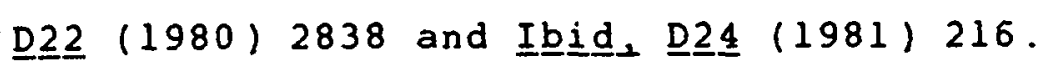

${ }^{10}$ C.E. DeTar, Phys.Rev_D2 4 (1981) 752 and_Iㅁiㅁ․, 762.

${ }^{11}$ R. Ayed, Rev.Mod.Phys. _ 2 , II, 2 (1981) S190-S191; Particle Data Group, I므므.

${ }^{12}$ R.A.Arndt_ett_al., Phys.Rev. _D2 0 (1979) 651 .

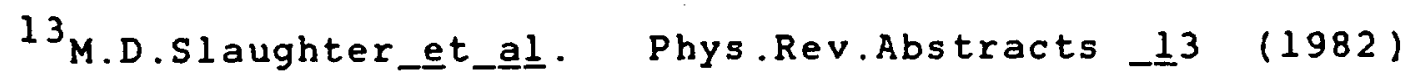
27 .

${ }^{14}$ T.D.Lee, Phys.Rev. _D15 (1977) 1694;_16 (1977) 1096 ;

1ㅇ. $(1978) 2673$.

${ }^{15}$ I.Duck and E. Umland, scheduled for publication in Phys . Lett.

${ }^{16}$ I.Duck and E. Umland, in preparation.

17 I므므.

${ }^{18}$ R.Goldflam, I. Duck, E. Umland, submitted to Phys. Lett. 


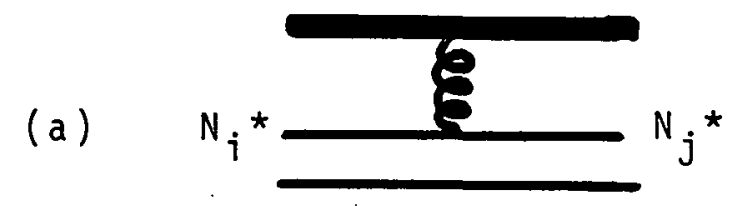

(b)

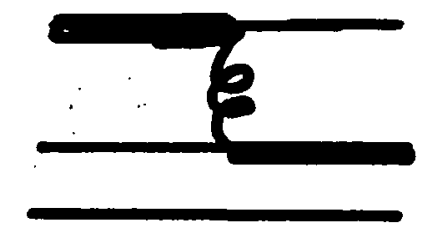

(c)

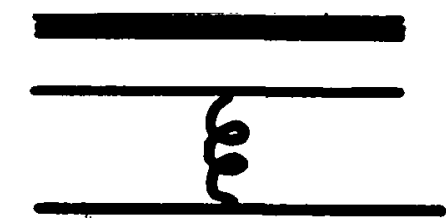

Figure 14

N* Self-Energies: Gluon Exchange

The bold bars are. $2 s$ quarks, the thin bars, $1 \mathrm{~s}$.

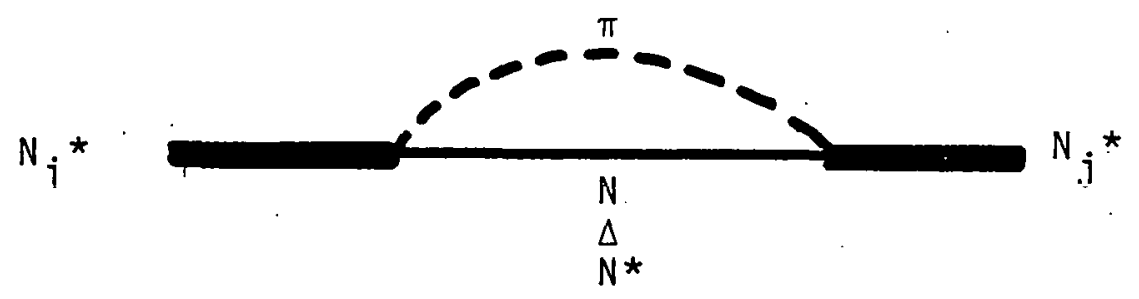

Figure 15

N* Self-Energ1es: Plon Radiative 


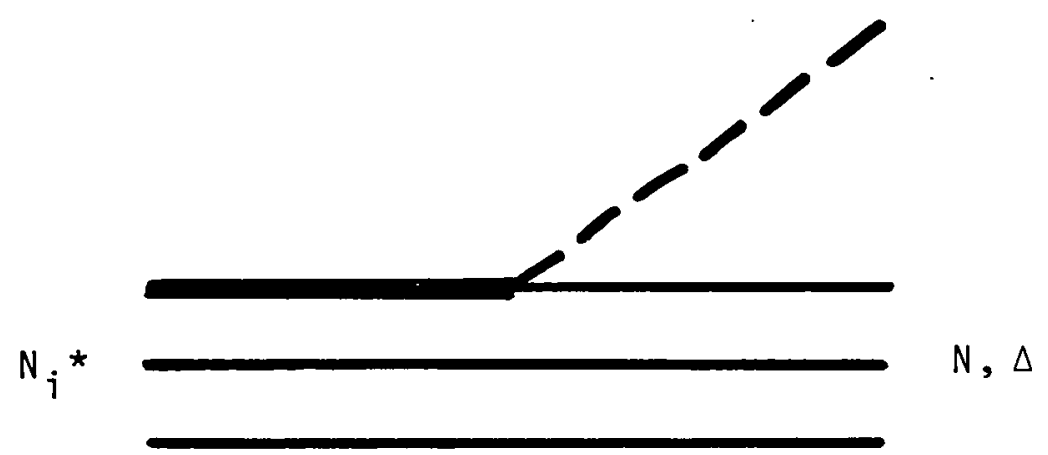

Figure 16

Zeroth-order $\mathrm{N}^{*}$ Baryon-Pion Coupling

The bold bars are $2 \mathrm{~s}$ quarks, the thin bars, $1 \mathrm{~s}$.

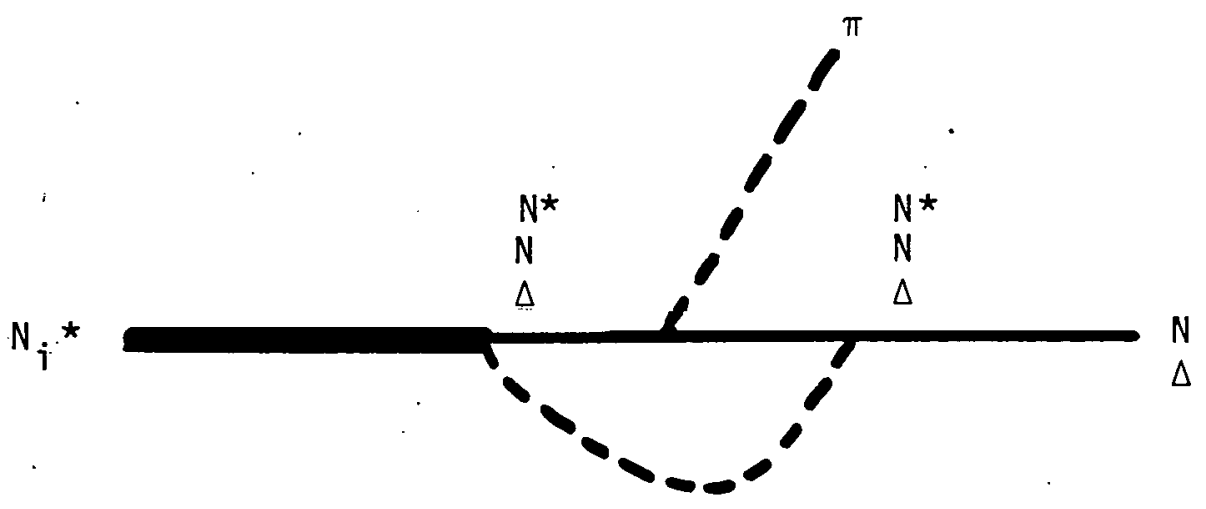

Figure 17

Pionic Radiative Vertex Corrections 
CHANGES IN PERSONNEI

FACULTY: S.D. Baker and G.S. Mutchler returned in August 1982, from their sabbaticals to begin academic year 1982-1983. Dr. Baker spent his leave working at Saturne, Saclay, and Dr. Mutchler worked with the University of Karlsrube Group at.S.I.N. in Switzerland. Their teaching duties were partially assumed during their absences by $D r$. B. E. Bonner from P-Division, Los Alamos National Laboratory. Dr. Bonner returned to his research at IAMPF in May 1982. In August $1982 \mathrm{G}$. S.Mutchler was promoted to full professor.

STAFF: Our technician of ten years, Joe Windish, died in March of 1982. To carry out some of his duties and projects, we are using the services of Jim Chappell, the Scientific Instrument Maker from the Space Physics support Shop here at Rice.

STUDENTS: J.D. Lesikar was awarded his Ph.D. degree : at the Rice Commencement in May 1982; Lt. Lesikar is currently serving as Corps Chemical officer at Fort sill, oklahoma: Charles Naudet is doing a High Energy Physics topic for his M.A. and joined this contract March 1, 1982. A new graduate student at Rice, Martin Marcin [M.A., University of Massachusetts ] also joined this contract. He is receiving 
his stipend support this academic year from Rice University.

OTHER EINANCIAI ASSISTANCE

No support external to Rice University and this contract for the work reported here occurred during the year except for stipend support from the National science Foundation to J.A. Rice [see budget attached].

\section{PUBIICATIONS}

1. M.M. Calkin, M.D.Corcoran, J.H.Hoftiezer, H.E.Miettinen, G.S.Mutchler, M.W.Arenton, D.S.Ayres, R.Diebold, R.Delzenero, E.N.May, I. Nodulman, J.Sauer, A.B.Wicklund, E.C.Swallow, "Density Matrix Elements of the Reaction p'p: $\Delta^{++}$n Between 1.2 and $2.0 \mathrm{GeV} / \mathrm{c}, "$ APS Bull. $\underline{2}$. (April 1982) 552 .

2. E.A.Umland, G.C.Phillips, G.S.Mutchler, J.B.Roberts, M.Duong-Van, "A High Sensitivity v-Detector

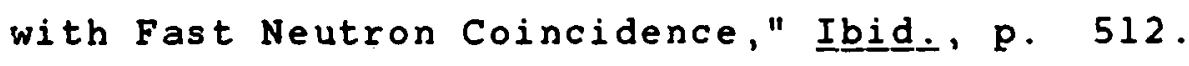

3. M.Duong-Van and P. Carruthers, "The Evidence for and Meaning of the Universality of Hadronic Multiplicities," I므르.; p. 457 .

4. M.Duong-Van, "The Hubble Constant in a Microscopic Universe," Ibid. p.544. 
5. M.D.Corcoran, M.M.Calkin, J.H.Hoftiezer, G.S. Mutchler, M.W.Arenton, D.S.Ayres, R.Diebold, E.N.May; L. Nodulman, J.R.Sauer, A.B.Wicklund, E.C. Swallow, "Analyzing Power in the Reaction ppod $\pi^{+}$for Beam Momenta from 1.17 to $1.96 \mathrm{GeV} / \mathrm{c}$, accepted by Physics Letters.

6. M.Arenton, R.Ditzler, T.Fields, M. Dris, M. Harrison, A.Kanofsky, R.Gustafson, L.Cormell, J.Fleischman, E.Gardella, C.Hitzman, W.Kononenko, B.Robinson, W.Selove, G.Theodosiou, B.Yost, M.Corcoran, K.Johns, H.Miettinen, C.Naudet, G.Phillips, J.Rice, J.Roberts, H.Chen, A.Erwin, A.Hasan, C.Kuehn, K.Nelson, and M.Thompson, "Evidence for Jets From a Transverse Energy Triggered Calorimeter Experiment at Fermilab," submitted to XXI High Energy Conference, Paris (July, 1982 ).

7. Argonne Fermilab Lehigh Michigan Pennsylvania Rice Wisconsin Collaboration, "E-609 at Fermilab: Study of High PT Hadron Collisions," U, of Pennsylvania Preprint UPR 100E ( June 25,1982 ).

Copies of the publications cited above are attached.

\section{INCIDENT REPORT}

No incidents such as those outlined in attachment "A" have occurred during the contract year.

October 1, 1982

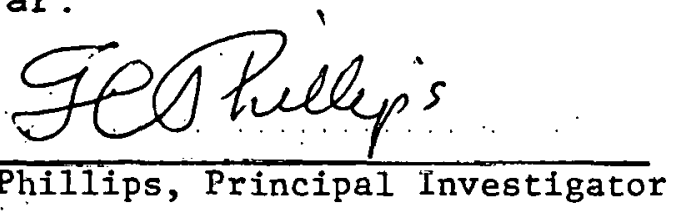


A P P E N D IX

PIBLICATIONS

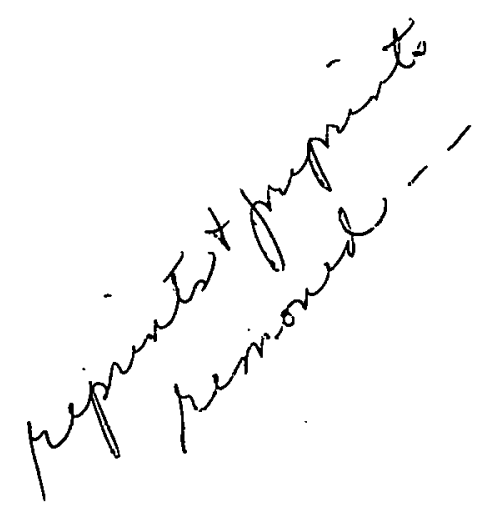

